Marina Pereira Ybarra Martins de Oliveira

\title{
Avaliação da prevalência e das características da síndrome dos ovários policísticos em adolescentes obesas
}

\author{
Dissertação apresentada à Faculdade de \\ Medicina da Universidade de São Paulo para \\ obtenção do título de Mestre em Ciências \\ Programa de Pediatria \\ Orientador: Prof. Dr. Durval Damiani
}

(Versão corrigida. Resolução CoPGr 5890, de 20 de dezembro de 2010.

A versão original está disponível na Biblioteca FMUSP)

SÃO PAULO

2016 
Dados Internacionais de Catalogação na Publicação (CIP)

Preparada pela Biblioteca da

Faculdade de Medicina da Universidade de São Paulo

Creprodução autorizada pelo autor

\section{Oliveira, Marina Pereira Ybarra Martins de}

Avaliação da prevalência e das características da síndrome dos ovários policísticos em adolescentes obesas / Marina Pereira Ybarra Martins de Oliveira. -- São Paulo, 2016.

Dissertação(mestrado)--Faculdade de Medicina da Universidade de São Paulo. Programa de Pediatria.

Orientador: Durval Damiani.

Descritores: 1.Adolescente 2.Obesidade 3.Prevalência 4.Ovário 5.Hiperandrogenismo 6.Ciclo menstrual 7.Síndrome do ovário policístico 8.Estudos transversais

USP/FM/DBD-141/16 


\section{DEDICATÓRIA}

Gostaria de dedicar esta dissertação ao meu melhor amigo, meu parceiro, meu marido. Aquele que sempre me apoia, me incentiva, me põe no eixo. Aquele que está sempre ao meu lado, me dando suporte para todos os desafios nos quais me proponho a enfrentar. Aquele que meu coração escolheu para amar a vida inteira. 


\section{AGRADECIMENTOS}

Gostaria de agradecer ao Professor Doutor Durval Damiani, meu orientador, por quem eu tenho uma admiração inenarrável e que manteve as portas abertas para eu pudesse "maternar" tranquilamente e me recebeu de volta, disponibilizando meu livre acesso como pesquisadora ao Serviço de Endocrinologia e, principalmente, ao setor de obesidade, pelo qual eu tenho grande afinidade, e foi base desta dissertação e do meu trabalho.

A todas as pacientes que concordaram em participar deste estudo. Sem elas nada disso seria possível.

A todos os assistentes da Endocrinologia Pediátrica do Instituto da Criança: Dra. Louise Cominato, Dra. Thais Della Manna, Dra. Leandra Steinmetz, Dr. Vaê Dichtcekennian, Dr. Hamilton Cabral Menezes-Filho, Dr. Hilton Kuperman e Dra. Nuvarte Setian, por terem me guiado durante a formação, me mostrando, com muito amor à profissão, diferentes caminhos a tomar.

À minha amiga e colega Dra. Ruth Rocha Franco, pela parceria diária, me ensinando, me apoiando e me ajudando nas pequenas e nas grandes decisões. E a todos os meus colegas, em especial a Dra. Caroline Passone, desse grupo em que a troca é mútua.

A Dra. Silvia Sucena, pela disponibilidade em realizar as ultrassonografias pélvicas e pela ajuda na redação dos termos técnicos, a Mariza Kazue, pelo apoio com a pesquisa bibliográfica, a Luis Stuginski, pelo banco de dados e pela disponibilidade e ao estatístico Lucas Damiani, pela paciência.

À Fundação de Amparo à Pesquisa do Estado de São Paulo (FAPESP) pelo auxílio financeiro deste trabalho (bolsa regular, número do processo 2104/11200-3). 
Aos meus pais, Justo e Cecília, pela base que me deram, por me apoiarem sempre e, em especial, a minha mãe pelo seu exemplo.

Aos meus filhos, Luke e $\mathrm{Pi}$, por fazerem brotar em mim um amor que eu desconhecia. 
"A vida é um recomeço contínuo numa eterna linha de mudança." (Eurípedes Constatino Miguel - psiquiatra e meu avô) 
Normalização adotada

\section{Padrão Vancouver}

Esta dissertação está de acordo com as seguintes normas, em vigor no momento desta publicação:

Referências: adaptado de International Committee of Medical Journals Editors (Vancouver).

Universidade de São Paulo. Faculdade de Medicina. Divisão de Biblioteca e Documentação. Guia de apresentação de dissertações, teses e monografias. Elaborado por Anneliese Carneiro da Cunha, Maria Julia de A. L. Freddi, Maria F. Crestana, Marinalva de Souza Aragão, Suely Campos Cardoso, Valéria Vilhena. 3a ed. São Paulo: Divisão de Biblioteca e Documentação; 2011.

Abreviaturas dos títulos dos periódicos de acordo com List of Journals Indexed in Index Medicus. 


\section{LISTA DE ABREVIATURAS E SIGLAS}

\section{LISTA DE TABELA}

LISTA DE FIGURAS

RESUMO

SUMMARY

1 INTRODUÇÃO

2 OBJETIVOS

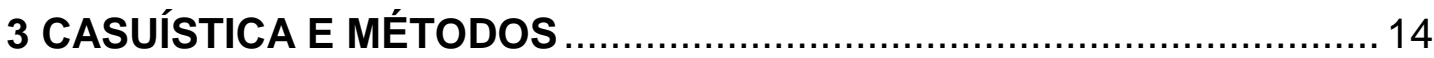

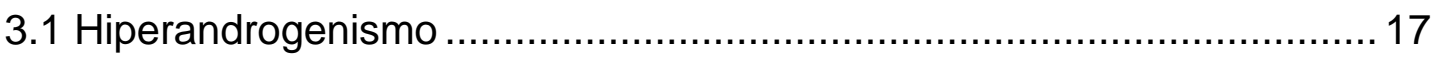

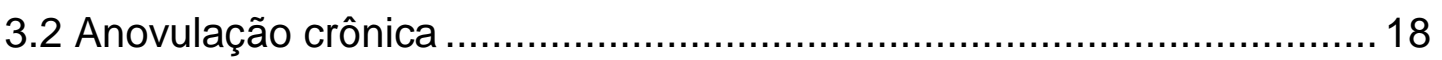

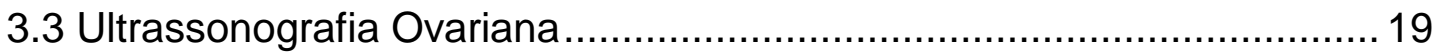

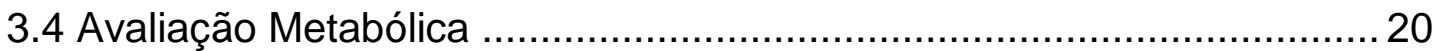

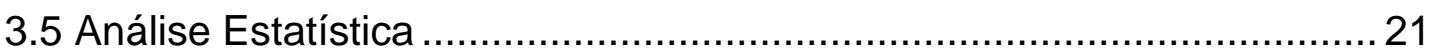

4 RESULTADOS

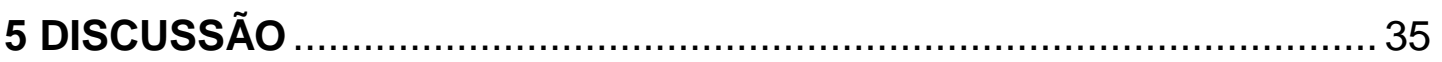

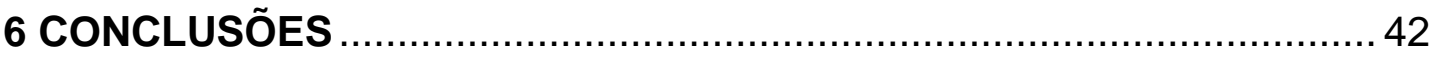

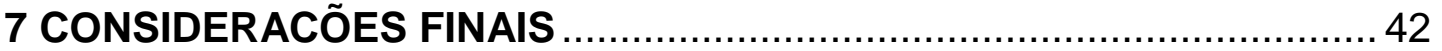

8 ANEXOS

9 REFERÊNCIAS 


\section{LISTA DE ABREVIATURAS E SIGLAS}

ACO - Anticoncepcionais Orais

AOcomSOP - Grupo de Adolescentes Obesas com Síndrome dos

AOsemSOP - $\quad \begin{aligned} & \text { Grupo de Adolescentes Obesas sem Síndrome dos } \\ & \text { Ovários Policísticos }\end{aligned}$

AOcomSM - Grupo de Adolescentes Obesas com Síndrome

Metabólica

AOsemSM - Grupo de Adolescentes Obesas sem Síndrome Metabólica

CA - Circunferência Abdominal

CA/A - Relação Circunferência Abdominal sobre a Altura

CAPPesq - Comissão de Ética para Análise de Projetos de Pesquisa

HPLC - Cromatografia Líquida de Alta Performance

DM-2 - Diabete Melito Tipo 2

IGF-I _ - Fator de Crescimento Insulínico Tipo I

F-G ～- Índice de Ferriman- Gallwey

GJ - Glicemia de Jejum

HAS - Hipertensão Arterial Sistêmica

TSH - Hormônio Estimulador da Tireoide

FSH - Hormônio Folículo Estimulante

LH $\quad$ - Hormônio Luteinizante

IAL $\quad-\quad$ Índice de Andrógenos Livres (IAL)

IMC - I Índice de Massa Corpórea

HOMA-IR - Índice Modelo de Avaliação Homeostático da Insulina

ICr - HC - Instituto da Criança do Hospital das Clínicas da

FMUSP - Faculdade de Medicina da Universidade de São Paulo

$\mathrm{NIH} \quad$ - Instituto Nacional de Saúde dos Estados Unidos da 
América

IOG - Intolerância Oral à Glicose

HDL - - Lipoproteína de Alta Densidade

NGSP - National Glycohemoglobin Standardization Program

n $\quad$ - Número de pacientes com a alteração

$\mathrm{N} \quad-\quad$ Número total de pacientes

OMS - Organização Mundial da Saúde

PIG - Pequeno para Idade Gestacional

PA - Pressão Arterial

SHBG - Proteína ligadora de hormônios sexuais

G/I - Relação Glicemia/Insulinemia de Jejum

RI - Resistência Insulínica

SI $\quad$ - Sensibilidade Insulínica

SOP $\quad$ - Síndrome dos Ovários Policísticos

SM - Síndrome Metabólica

SEP - Sociedade de Endocrinologia Pediátrica

AES Sociedade de Excesso Androgênico e Síndrome dos Ovários Policísticos

S\&P $\quad-\quad$ Sultan \& Paris

$\mathrm{T}$

- Tempo

TCLE - - Termo de Consentimento Livre e Esclarecido

TOTG - Teste Oral de Tolerância à Glicose

USG - Ultrassonografia 


\section{LISTA DE TABELA}

Tabela 1 - Estatísticas descritivas das variáveis contínuas 23

Tabela 2 - Prevalências das variáveis utilizadas para diagnosticar síndrome dos ovários policísticos.

Tabela 3 - Análise da relação do hiperandrogenismo clínico aferido por meio do hirsutismo pelo índice de Ferriman e Gallwey $>$ ou $=8$, com 0 hiperandrogenismo laboratorial. 26

Tabela 4 - Prevalências de Síndrome dos Ovários Policísticos 27

Tabela 5 - Comparação das variáveis contínuas segundo a Síndrome dos Ovários Policísticos (SOP) pelo critério da Diretriz de SOP na adolescência segundo a Sociedade de Endocrinologia Pediátrica americana

Tabela 6 - Proporção de uso de anticoncepcional e metformina segundo a Síndrome dos Ovários Policísticos (SOP), estabelecida pelo critério da Diretriz de SOP na adolescência segundo a Sociedade de Endocrinologia Pediátrica americana. 31

Tabela 7 - Distribuição dos critérios de diagnóstico de Síndrome dos Ovários Policísticos segundo a presença de Síndrome Metabólica 


\section{LISTA DE FIGURAS}

Figura 1. Resumo dos critérios diagnósticos da Síndrome dos Ovários Policísticos para mulheres adultas. .............................................................. 7

Figura 2. Resumo dos critérios diagnósticos da Síndrome dos Ovários Policísticos para adolescentes. .................................................................... 10

Figura 3. Fluxograma da amostra selecionada, com os motivos pelos quais 36 pacientes foram excluídas do estudo....................................................... 16

Figura 4. Índice semiquantitativo de Ferriman e Gallwey modificado para avaliação de hirsutismo(8)................................................................... 18 


\section{RESUMO}

Oliveira MPYM. Avaliação da prevalência e das características da síndrome dos ovários policísticos em adolescentes obesas [Dissertação]. São Paulo: Faculdade de Medicina, Universidade de São Paulo; 2016.

Introdução: o diagnóstico da Síndrome do Ovário Policístico (SOP) na adolescência é desafiador e vem sendo alvo de intensas discussões. Sua prevalência em mulheres adultas em idade fértil varia de 5-10\%. Entretanto, a prevalência em adolescentes obesas ainda não foi descrita na literatura. Somado a isso, ainda não é bem estabelecida a relação da SOP com alterações metabólicas e cardiovasculares nesta população. Dessa maneira, objetivamos avaliar a prevalência e as características da SOP na população de adolescentes obesas acompanhadas em um centro hospitalar quaternário.

Métodos: realizamos um estudo transversal com 49 adolescentes obesas pósmenarca, com idade média de 15,6 anos. Foi realizada avaliação antropométrica e revisão de prontuários médicos. O hiperandrogenismo clínico e laboratorial foram quantificados utilizando o índice de Ferriman-Gallwey e as dosagens androgênicas, respectivamente. A morfologia ovariana foi avaliada por ultrassonografia suprapúbica. Todas as pacientes tiveram seus perfis metabólicos analisados. Resultados: ao adotarmos a nova Diretriz para SOP na adolescência da Sociedade de Endocrinologia Pediátrica Americana, encontramos uma prevalência de $18,4 \%$ de SOP em nossa população de adolescentes obesas. Quando utilizamos os critérios de Rotterdam, da Sociedade de Excesso Androgênico e SOP e do Instituto Nacional de Saúde Americano, as prevalências foram de $26,4 \%, 22,4 \%$ e $20,4 \%$, respectivamente. A irregularidade menstrual foi constatada em $65,3 \%$ das pacientes. $O$ hiperandrogenismo clínico foi observado em $16,3 \%$ das meninas, e $18,4 \%$ tinham concentrações de testosterona total acima do valor de normalidade. A ultrassonografia revelou que $18,4 \%$ das meninas tinham ovários policísticos. Adolescentes obesas com SOP apresentaram maior prevalência de síndrome metabólica. Conclusão: a prevalência de SOP em adolescentes obesas é alta quando comparada àquela observada na literatura. 
Descritores: Adolescente; Obesidade; Prevalência; Ovário; Hiperandrogenismo; Ciclo Menstrual; Síndrome do Ovário Policístico; Estudos Transversais. 


\section{SUMMARY}

Oliveira MPYM. Prevalence and characteristics of polycystic ovary syndrome in obese adolescents [Dissertation]. São Paulo: Faculdade de Medicina, Universidade de São Paulo; 2016.

Background: polycystic ovary syndrome (PCOS) in adolescence is a challenging diagnosis and therefore has raised intense discussions. Its prevalence in childbearing age women ranges from 5 to $10 \%$. However, the prevalence in obese adolescents has not yet been reported. Besides, the relationship of PCOS with metabolic and cardiovascular disorders in this specific population has not been established. Thus, we aimed to assess the prevalence and characteristics of PCOS in a population of obese adolescents followed at a quarternary hospital. Methods: we performed a cross-sectional study with 49 postmenarcheal obese adolescents with a mean age of 15.6 years. Anthropometric assessment and review of medical records were performed. Clinical and laboratory hyperandrogenism were evaluated using Ferriman-Gallwey index and serum androgens, respectively. The ovarian morphology was evaluated by supra-pubic ultrasound. All patients had their metabolic profile evaluated. Results: the prevalence of PCOS in obese adolescents, according to the new guideline for PCOS in adolescence of the American Pediatric Endocrinology Society, was $18.4 \%$. When assessed by the Rotterdam, the Androgen Excess and PCOS Society and the National Institute of Health criteria, the prevalence of PCOS was $26.4 \%, 22.4 \%$ and $20.4 \%$, respectively. Menstrual irregularity was found in $65.3 \%$ of the patients. Clinical hyperandrogenism was observed in $16.3 \%$ while $18.4 \%$ had total testosterone concentrations above the normal range. Ultrasonography revealed that $18.4 \%$ had polycystic ovaries. Obese adolescents with PCOS had higher prevalence of metabolic syndrome. Conclusion: the prevalence of PCOS in obese adolescents is high compared to that observed in the literature.

Descriptors: Adolescent; Obesity; Prevalence; Ovarian; Hyperandrogenism; Menstrual Cycle; Polycystic Ovary Syndrome; CrossSectional Studies. 
1 INTRODUÇÃO 
A Síndrome dos Ovários Policísticos (SOP) foi descrita em relatos médicos desde a época de Hipócrates. Em 1935, Stein \& Leventhal descreveram sete casos de mulheres com amenorreia e hirsutismo associados a ovários aumentados e policísticos, originando o nome da síndrome na década de 1960. ${ }^{(1)}$ Trata-se do distúrbio endocrinológico mais comum em mulheres em idade fértil (5-10\%) e a principal causa de infertilidade feminina. ${ }^{(2)}$ Estima-se que, no mundo, 105 milhões de mulheres entre 15 e 49 anos de idade apresentem SOP e que esta seja responsável por $72-82 \%$ das causas de hiperandrogenismo. ${ }^{(3)}$ É uma doença de difícil diagnóstico, pois se apresenta de forma heterogênea, cujos sinais e sintomas mudam ao longo da vida, sem nenhum exame que defina claramente o diagnóstico.

Uma das hipóteses para a etiologia da SOP é que a exposição androgênica intraútero, devido a fatores genéticos e/ou ambientais, sensibilizaria o ovário fetal, o qual durante o estímulo hormonal da puberdade reagiria com uma hiperprodução androgênica, desencadeando a SOP. Tal hipótese surgiu em estudos com crias de macacas Rhesus que, após terem sido expostas a uma superdosagem de testosterona na vida embrionária, desenvolveram características semelhantes às da SOP, tais como: hipersecreção de hormônio luteinizante (LH), hiperandrogenismo ovariano, anovulação, aumento do índice de massa corpórea (IMC) e resistência insulínica (RI). ${ }^{(4)}$

A patogenia da SOP ainda não foi bem elucidada e envolve fatores genéticos e ambientais, assim como contribuições hereditárias e não hereditárias. A herança poligênica parece ser responsável por $70 \%$ da heterogeneidade de sua apresentação clínica e laboratorial. Recentemente, um polimorfismo no promotor $-1031(\mathrm{~T} / \mathrm{C})$ do fator de necrose tumoral-alfa foi relacionado à SOP. ${ }^{(5)}$ História familiar de SOP, de ovários policísticos, de obesidade central, de diabetes mellitus tipo 2 (DM-2) ou outros aspectos de RI podem conferir maior risco de SOP. Os ovários policísticos parecem ter 
transmissão autossômica dominante e, geralmente, não produzem sintomas, mas podem estar associados à SOP, com disfunção ovariana subclínica. ${ }^{(6)}$

De qualquer forma, mesmo tendo sua origem na vida fetal, a SOP costuma manifestar-se clinicamente somente na vida adulta, após a maturação do eixo hipotálamo-hipófise-gonadal, quando a secreção exagerada de LH leva a uma maior produção androgênica ovariana.

A síndrome é caracterizada pelas altas concentrações de LH em relação ao hormônio folículo estimulante (FSH) (50-75\%), resultando no aumento da produção de andrógenos pelas células da teca. ${ }^{(7)}$ Esses níveis aumentados de andrógenos influenciam o feedback da progesterona e do estradiol, contribuindo para a manutenção dos altos níveis de LH circulantes e perpetuando o ciclo. A testosterona é o principal andrógeno circulante na mulher com SOP e, apesar de sua concentração total poder estar na faixa da normalidade, sua biodisponibilidade pode encontrar-se aumentada devido aos baixos níveis de proteína ligadora de hormônios sexuais (SHBG). ${ }^{(7)}$

A hiperandrogenemia se manifesta clinicamente por meio de três sinais característicos da SOP: acne, alopecia androgênica e hirsutismo, este último aferido pelo índice semiquantitativo de Ferriman-Gallwey (F-G) ${ }^{(8)}$ (Figura 2). $\mathrm{O}$ valor maior ou igual a 8 para se definir que uma paciente é hirsuta foi estabelecido em mulheres de etnia branca e adultas (18-38 anos). ${ }^{(9)}$

A RI e a hiperinsulinemia também estão inseridas na fisiopatologia da SOP $(44-70 \%){ }^{(10,11)}$ Altas concentrações de insulina são capazes de estimular a produção do fator de crescimento insulínico tipo I (IGF-I) e ativar receptores localizados nas células da teca, estimulando a esteroidogênese ovariana com consequente hiperandrogenemia. Além disso, a hiperinsulinemia diminui a síntese hepática de SHBG, aumentando a biodisponibilidade androgênica. ${ }^{(12)}$

$\mathrm{Na}$ ultrassonografia, a semelhança visual dos folículos com uma imagem cística levou ao nome policístico, que nada mais é do que multifolicular. A presença de cistos/folículos ovarianos somente deve ser considerada patológica quando estiver em número aumentado, sendo a SOP sua principal causa. Para o diagnóstico de ovários policísticos, em mulheres adultas, a contagem de folículos parece ser um bom parâmetro. ${ }^{(13)}$ A quantidade de folículos que determina a presença de SOP é divergente na literatura, e o valor 
do limite inferior varia entre 10 e 12 . No entanto, recentemente uma diretriz estabeleceu que o valor de corte deva ser de mais de 25 folículos em cada ovário visto pela ultrassonografia pélvica via transvaginal, com transdutor com frequência $>8 \mathrm{MHz}$. ${ }^{(13)}$

A obesidade é também um diagnóstico muito frequentemente associado à SOP. Estima-se que 35 a $60 \%$ das mulheres adultas com SOP sejam obesas. ${ }^{(14)}$ O tecido adiposo é o maior sítio de conversão dos precursores androgênicos ${ }^{(15)}$ e o aumento da adiposidade está associado com a diminuição de SHBG e com hiperandrogenemia. ${ }^{(16)}$ A obesidade é uma doença inflamatória que cursa com RI periférica e consequente hiperinsulinemia. A correção ou supressão da hiperinsulinemia, por meio da perda de peso ${ }^{(17)}$ ou do tratamento medicamentoso, ${ }^{(18)}$ leva à diminuição das concentrações androgênicas e melhora da função ovariana, evidenciada pela regulação dos ciclos menstruais e do status ovulatório. ${ }^{(10,11,19,20)}$ A diminuição de pelo menos $5 \%$ no peso corporal de mulheres com SOP pode levar a uma melhora de seus índices metabólicos e de sua fertilidade. ${ }^{(4)}$

Além da alta prevalência de infertilidade observada nas pacientes com SOP, elas compartilham fatores de risco associados ao desenvolvimento de câncer endometrial. Há também associação com hipertensão arterial, apneia obstrutiva do sono, redução da fibrinólise e da vasodilatação, todos levando a um maior risco cardiovascular. ${ }^{(21)}$ Foi também relatada maior incidência de depressão e transtornos de ansiedade. ${ }^{(21)}$

$\mathrm{Na}$ adolescência, a fisiopatologia da SOP fica ainda mais difícil de ser elucidada, uma vez que as alterações encontradas na SOP se confundem com alterações fisiológicas próprias dessa fase. Durante esse período, há diminuição da sensibilidade insulínica (SI) e hiperinsulinemia compensatórias. Isso desencadeia um aumento transitório da produção de hormônio do crescimento e diminuição da SHBG. Um estudo mostrou que as dosagens androgênicas parecem ser mais elevadas em adolescentes saudáveis do que em mulheres adultas. ${ }^{(22)}$

Os ciclos menstruais podem ser irregulares e anovulatórios nos primeiros anos depois da menarca (anovulação fisiológica da adolescência), podendo persistir por vários anos ${ }^{(23,24)}$ e, não necessariamente, correlacionar- 
se com sinais de hiperandrogenismo. ${ }^{(25)}$ No primeiro ano depois da menarca, $75 \%$ das meninas apresentam ciclos regulares (entre 21-45 dias), dos quais cerca da metade deles são anovulatórios, apesar do ritmo regular. ${ }^{(26)}$ Nesse contexto, foi sugerido que se aguardasse pelo menos dois anos depois da menarca para se determinar que a irregularidade menstrual está de fato presente. ${ }^{(27)} \mathrm{A}$ amenorreia primária é uma manifestação incomum de SOP, variando de 1,4 a $14 \%{ }^{(28)}$

Acredita-se que o principal mecanismo do hiperandrogenismo da SOP seja ovariano, embora, na adolescência, a adrenal possa ter um papel importante nessa secreção, podendo levar à acne e ao hirsutismo leves. ${ }^{(7)} \mathrm{A}$ acne é comumente vista nessa fase e não pode ser considerada como critério clínico de hiperandrogenismo, ao mesmo tempo em que a alopecia androgênica dificilmente se instala nessa faixa etária, restando apenas o hirsutismo a ser valorizado como parâmetro clínico de hiperandrogenismo. ${ }^{(6,29 \text {, }}$ ${ }^{30)}$ A causa mais comum de hirsutismo na adolescência é a SOP. ${ }^{(31)}$

Entretanto, não existem valores de corte para a população adolescente embora já tenha sido sugerido que utilizassem valores inferiores aos propostos para a população adulta. ${ }^{(21,25,27,32,33)}$

Durante a adolescência, o número de folículos está fisiologicamente aumentado. ${ }^{(34,35)}$ Foi demonstrado que até $8 \%$ das adolescentes apresentam imagem de ovários policísticos associados a ciclos menstruais irregulares, contudo, sem evidência clínica ou laboratorial de excesso androgênico. É alarmante que em $42 \%$ dessas adolescentes já se verifica disfunção ovariana. ${ }^{(6)}$

$\mathrm{Na}$ adolescência, o volume ovariano é sugerido como melhor parâmetro para o diagnóstico de ovários policísticos, apesar de, durante esse período, haver uma variação em que o volume ovariano máximo ocorre nos primeiros anos depois da menarca e diminui progressivamente até a vida adulta. ${ }^{(36)}$ Nessa fase, a ultrassonografia é realizada via suprapúbica, o que diminui a resolução e a acurácia da verificação do número de folículos, especialmente em adolescentes obesas. ${ }^{(35)}$ Apesar de Fruzzetti et al. demonstrarem que a ultrassonografia pélvica via suprapúbica apresenta baixa sensibilidade como critério diagnóstico de SOP na adolescência, ${ }^{(37)}$ o volume ovariano maior de 10 
$\mathrm{mL}$, dois anos após a menarca, é um valor de corte bem aceito pela maioria dos autores. ${ }^{(13,36-40)}$ Os achados radiológicos podem ser mais bem interpretados quando associados a sinais clínicos e laboratoriais consistentes com SOP. ${ }^{(41)}$

É descrita uma associação entre crianças que nasceram pequenas para idade gestacional (PIG) e a pubarca precoce. ${ }^{(42)}$ Há também uma associação entre PIG e a oligomenorreia. ${ }^{(43)}$ Tanto a adrenarca como a pubarca precoces (15-20\%) estão associadas a maior risco de SOP na adolescência, ${ }^{(4)}$ principalmente a adrenarca exagerada. ${ }^{(6)}$

A recente epidemia de obesidade na infância traz diversas preocupações sobre seus efeitos de curto e longo prazos, incluindo o aumento da prevalência e da gravidade dos sintomas de SOP na adolescência. A hiperandrogenemia, muitas vezes motivada por excesso de LH, preserva um quadro clínico de SOP e, além disso, a hiperinsulinemia associada à obesidade (30-75\%) pode resultar na progressão da SOP em meninas susceptíveis. ${ }^{(6,44)}$

A obesidade na adolescência pode levar a sintomas de hiperandrogenismo e anovulação, como hirsutismo, acne persistente e irregularidade menstrual. Lewy et al. mostraram que meninas obesas com SOP apresentam concentrações duas vezes maiores de insulinemia de jejum e SI periférica $50 \%$ menor do que as controles obesas sem SOP. ${ }^{(20)}$

O DM-2, reconhecido na juventude no começo dos anos 1980, é considerado raro na população pediátrica. No entanto, tem ocorrido uma tendência perturbadora de aumento dos casos de DM-2 relacionados à obesidade, especialmente em meninas com hiperandrogenismo, ciclos menstruais irregulares e Acanthosis nigricans. ${ }^{(45,46)}$ Meninas com SOP e excesso de peso têm de três a dez vezes mais chance de desenvolver DM-2 na vida adulta (5-10\%) e um risco duas vezes maior de desenvolver síndrome metabólica (SM). ${ }^{(15)}$

A dislipidemia também está frequentemente associada à SOP. Ojaniemi et al. mostraram que tanto a dislipidemia como a obesidade são mais frequentemente associadas à SOP na adolescência do que na vida adulta, sendo que mais da metade das adolescentes com SOP apresentavam um perfil lipídico mais aterogênico, com níveis de lipoproteína de alta densidade (HDL) 
abaixo do esperado para a idade, e níveis aumentados de triglicérides, o que leva a acreditar que exista um aumento do risco cardíaco nessa população. ${ }^{(15)}$

Tamanha heterogeneidade de sinais e sintomas, assim como a complexidade de sua fisiopatologia se refletem nos diferentes critérios diagnósticos propostos para a SOP.

O Instituto Nacional de Saúde dos Estados Unidos da América (NIH), em 1990, estabeleceu como critérios diagnósticos da SOP a necessidade da presença tanto de irregularidade menstrual como de excesso androgênico. ${ }^{(47)}$

\section{CRITÉRIOS}

Rotterdam
(2 de 3$)$

AES

Hiperandrogenismo

Clínico ou Laboratorial

Anovulação Crônica ou

Irregularidade Menstrual $\operatorname{sim}$

e

e/ou

$\operatorname{sim}$

$\operatorname{sim}$

$\operatorname{sim}$

e/ou

e/ou

\section{Ovários Policísticos}

$$
\text { ou }>10 \mathrm{~mL}
$$

$\operatorname{sim}$

$\operatorname{sim}$

Figura 1. Resumo dos critérios diagnósticos da Síndrome dos Ovários Policísticos para mulheres adultas. NIH: Instituto Nacional de Saúde dos Estados Unidos da América; Rotterdam: Criétios de Rotterdam; AES: Sociedade de Excesso Androgênico e Síndrome dos Ovários Policísticos; ${ }^{(47-49)}$

A Sociedade Europeia de Reprodução Humana e Embriologia, em conjunto com a Associação Americana de Medicina Reprodutiva, em 2003 e revisados em 2004 (Rotterdam), não concordaram com a exclusão dos ovários policísticos dos critérios diagnósticos e estabeleceram que seriam necessárias a presença de duas das três características que se seguem: sinais de 
anovulação crônica por mais de seis meses; sinais de hiperandrogenismo clínicos (hirsutismo, acne ou alopecia androgênica) ou laboratorial; ou ovários policísticos diagnosticados pelo exame ultrassonográfico (volume ovariano > $10 \mathrm{~mL}$ ou $\geq 12$ folículos entre $2-9 \mathrm{~mm}$ ). ${ }^{(48)}$ Isso permitiu que se fizesse 0 diagnóstico de SOP sem a presença de hiperandrogenismo, considerado por muitos o pilar da síndrome. Então, em 2009, a Sociedade de Excesso Androgênico e SOP (AES) instituiu que, para se fazer o diagnóstico de SOP, seria obrigatória a presença de hiperandrogenismo, que deveria estar associado à disfunção ovariana (irregularidade menstrual e/ou a ovários policísticos) (Tabela 1). ${ }^{(49)}$ Todos os critérios entendem a SOP como um diagnóstico de exclusão e exigem que antes de se estabelecer esse diagnóstico devam ser descartados: hiperplasia congênita das suprarrenais forma não clássica, síndrome de Cushing, disfunção tireoidiana e/ou tumores secretores de andrógenos.

Recentemente, a Sociedade de Endocrinologia Pediátrica americana (SEP) reuniu especialistas em endocrinologia pediátrica e adolescência do mundo inteiro e publicou uma diretriz para o diagnóstico de SOP na adolescência (Diretriz-SEP), o qual sugere que, para se estabelecer o diagnóstico de SOP na adolescência, há necessidade tanto da presença de hiperandrogenismo clínico ou laboratorial como de irregularidade menstrual. Sendo o hiperandrogenismo clínico diagnosticado na presença de hirsutismo moderado a grave, de acordo com a etnia da população estudada, sem estabelecer valor de corte do F-G. A presença de acne persistente e pouco responsiva a tratamento tópico indicaria a investigação de hiperandrogenismo laboratorial. O hiperandrogenismo laboratorial é o melhor critério diagnóstico de SOP na adolescência e está presente quando as concentrações de testosterona total e/ou livre estiverem maiores do que os valores de referência do método utilizado. A irregularidade menstrual foi definida como ciclos menstruais menores de vinte dias ou maiores de quarenta e cinco dias pelo menos dois anos depois da menarca; ciclos maiores que 90 dias; ou ausência de menstruação aos 15 anos de idade ou 2-3 anos após a telarca. A presença de ovários com volume maior que $12 \mathrm{~mL}$, vistos na ultrassonografia, deveria ser considerada apenas como suspeita de SOP quando associada a outros 
sinais. Considera-se que a ultrassonografia via suprapúbica, especialmente em meninas obesas, pode trazer informações não confiáveis. E recomendou 0 tratamento específico para alívio dos sintomas, independente de se estabelecer o diagnóstico de certeza. ${ }^{(50)}$

Antes disso, houve algumas tentativas de estabelecimento de critérios diagnósticos para a adolescência. Sultan \& Paris, em 2006, incluíram a hiperinsulinemia nos critérios diagnósticos e sugeriram a necessidade da presença de quatro dos cinco critérios a seguir: oligomenorreia ou amenorreia pelo menos dois anos após a menarca; hiperandrogenismo clínico; hiperandrogenismo laboratorial; RI ou hiperinsulinemia; ovários policísticos (S\&P). ${ }^{(51)}$ Carmina et al., em 2010, propuseram que, para se estabelecer 0 diagnóstico de SOP em adolescentes, as meninas precisariam apresentar três dos seguintes sinais: hiperandrogenismo laboratorial (testosterona total elevada), oligomenorreia e volume ovariano maior que $10 \mathrm{~mL}$ (Tabela 2). ${ }^{(39)}$

Embora seja uma das endocrinopatias mais comuns nas mulheres em idade reprodutiva, a SOP não costuma ser um diagnóstico investigado na idade pediátrica, talvez porque a fertilidade ainda não seja uma preocupação nesta faixa etária. A prevalência de SOP na adolescência parece ser menor do que na população adulta, entretanto, meninas obesas predispostas ou já com diagnóstico de SOP podem apresentar manifestações clínicas mais importantes e precoces. ${ }^{(52)} \mathrm{Em}$ adolescentes obesas, a prevalência de SOP ainda não está bem estabelecida, já que seu diagnóstico pode ser mascarado pelas similaridades entre as mudanças fisiológicas da puberdade e as alterações da SOP, especialmente quando associadas à obesidade.

Estabelecer o diagnóstico da SOP na adolescência, ou até antes disso, permitiria não somente identificar a doença, como também realizar o tratamento precoce da SOP e de suas complicações, possibilitando uma eventual redução da morbidade e aumento das taxas de fertilidade na vida adulta. ${ }^{(53-55)}$ A hipótese deste estudo é que a prevalência de SOP em adolescentes obesas seja alta, suficiente para justificar uma investigação ativa nestas pacientes. 
CRITÉRIOS

\section{$S \& P$ \\ (4 de 5) Carmina et al Diretriz-SEP}

Hiperandrogenismo

Clínico

$\operatorname{sim}$

$-$

e/ou

$\operatorname{sim} \quad$ Testo T alta

e/ou

e

$\operatorname{sim}$

Anovulação Crônica ou
Irregularidade Menstrual*

e/ou

e

Podendo ou não

estar associado a moderado a grave

e/ou

Testo $\mathrm{T} / \mathrm{L}$ altas

e

$\operatorname{sim}$

Ultrassonografia Ovariana $>10 \mathrm{~mL} \quad>10 \mathrm{~mL} \quad>12 \mathrm{~mL}^{* *}$

e/ou

Resistência Insulínica ou Hiperinsulinemia

$\operatorname{sim}$

Figura 2. Resumo dos critérios diagnósticos da Síndrome dos Ovários Policísticos (SOP) para adolescentes. S\&P: Sultan \& Paris; Diretriz-SEP: diretriz de SOP na adolescência da Sociedade de Endocrinologia Pediátrica americana; Testo T alta: concentrações de testosterona total acima dos limites de normalidade do método utilizado; Testo T/L altas: concentrações de testosterona total e/ou livre acima dos limites de normalidade do método utilizado. *Inclui amenorreia primária aos 15 anos ou 2-3 anos após telarca. **Não é considerada como critério diagnóstico, porém, se alterado, sugere-se investigação para SOP. ${ }^{(21,39,50,51)}$ 
2 OBJETIVOS 
O objetivo deste estudo foi verificar a prevalência da SOP em adolescentes obesas, de acordo com a Diretriz-SEP(50), e compará-la a outros diferentes critérios diagnósticos propostos na literatura. ${ }^{(47-49)}$

Secundariamente, realizamos uma avaliação clínica e metabólica do grupo de adolescentes obesas com SOP (AOcomSOP) e sem SOP (AOsemSOP) diagnosticadas pelo critério da Diretriz-SEP. 
3 CASUÍSTICA E MÉTODOS 
O presente estudo foi aprovado pelo Comitê de Ética em Pesquisa do Departamento de Pediatria do Instituto da Criança do Hospital das Clínicas da Faculdade de Medicina da Universidade de São Paulo (ICr - FMUSP) e pela Comissão de Ética para Análise de Projetos de Pesquisa - CAPPesq, número 11446 em janeiro de 2014 (Anexo A). Todas as pacientes incluídas e seus responsáveis legais concordaram em participar do estudo e assinaram o Termo de Consentimento Livre e Esclarecido (TCLE), versão 2, de 01/11/2013 (Anexo B).

Adotamos os seguintes critérios de inclusão e exclusão:

Critérios de inclusão- pacientes do sexo feminino; idade entre 10 e 18 anos; matriculadas no Ambulatório de Obesidade da Unidade de Endocrinologia Pediátrica do Departamento de Pediatria do ICr-FMUSP; diagnóstico de sobrepeso $(Z$ score $>+1)$, obesidade $(Z$ score $>+2)$ ou obesidade grave ( $Z$ score $>+3$ ), definidos por meio da medida do IMC (peso em quilogramas dividido pela estatura em metros elevada ao quadrado) para idade e sexo da curva da Organização Mundial da Saúde.

Critérios de exclusão: não ter apresentado menarca ou ter apresentado menarca há menos de dois anos da data do estudo; gestação; ter realizado cirurgia bariátrica; hipotireoidismo não controlado; hiperplasia congênita das suprarrenais - forma não clássica; síndrome de Cushing; tumores secretores de andrógenos; hiperprolactinemia; não ter assinado ou entregue o TCLE. 
Avaliadas entre $2014-2015$

n: 85

\section{$\mathrm{n}: 49$ pacientes}

Figura 3. Fluxograma da amostra selecionada, com os motivos pelos quais 36 pacientes foram excluídas do estudo.

No período do estudo, durante os anos de 2014 e 2015, compareceram ao serviço 85 pacientes. Destas, 49 foram avaliadas e incluídas no presente estudo. As demais, 36, foram excluídas devido aos seguintes motivos: não aceitou participar $(n=1)$; não entregou termo de consentimento livre e esclarecido assinado pelo responsável legal $(n=2)$; gestação durante a avaliação $(n=1)$; não apresentou menarca ou apresentou há menos de 2 anos do estudo ( $n=18)$; havia realizado gastrectomia vertical laparoscópica para tratamento da obesidade $(n=10)$; e não completou a investigação diagnóstica $(n=4)$ (Figura 1). Não houveram casos de meninas com diagnóstico de amenorreia primária.

Durante a investigação laboratorial, todas as pacientes fizeram o teste de gravidez por meio da dosagem sérica de beta-HCG (Eletroquimioluminescência, RocheDiagnostics, Mannheim, Germany, $\mathrm{mUl} / \mathrm{mL}$ ). Afastaram-se a presença de hiperplasia congênita das suprarrenais - forma não clássica, por meio de concentrações de 17-OH-Progesterona < 200ng/dL ${ }^{(56)}$ (Radioimunoensaio Beckman Coulter, Czech Republic, ng/mL), hiperprolactinemia (Eletroquimioluminométrico RocheDiagnostics, Mannheim, Germany, ng/mL), síndrome de Cushing por meio da avaliação clínica e da 
dosagem sérica de cortisol basal (Quimioimunoensaio Siemens, Malvern, PA, $\mathrm{ug} / \mathrm{dL}$ ) e hipotireoidismo por meio das concentrações normais do hormônio estimulador da tireoide (TSH) (Eletroquimioluminométrico RocheDiagnostics, Mannheim, Germany, ulU/mL).

A SOP foi investigada pelos vários critérios descritos anteriormente: $\mathrm{NIH}$, Rotterdam, AES, Diretriz-SEP, S\&P e Carmina et al.

Os dados foram coletados durante as consultas de rotina do ambulatório e por meio da revisão de prontuários médicos. As seguintes características clínicas e demográficas foram registradas: idade; etnia; história familiar positiva (história familiar de SOP, ou obesidade, ou SM, ou doenças cardiovasculares); peso e comprimento ao nascer, sendo considerados PIG pacientes com peso de nascimento $<2500 \mathrm{~g}$ ou comprimento ao nascer $<47 \mathrm{~cm}$; ${ }^{(57)}$ uso de metformina e de anticoncepcionais orais (ACO). A avaliação laboratorial foi realizada durante a coleta regular de exames das pacientes no ambulatório em momento aleatório do ciclo menstrual.

\subsection{Hiperandrogenismo}

Para avaliação do hiperandrogenismo clínico, pesquisou-se a presença de hirsutismo segundo o índice semiquantitativo de Ferriman-Gallwey (F-G) (Figura 2$)^{(8)}$, e adotou-se o valor de corte $\geq 8{ }^{(3,58)}$ Também foram avaliadas a presença de acne (leve, moderada ou grave) e de alopecia androgênica. $O$ hiperandrogenismo laboratorial foi considerado para as concentrações de testosterona livre $>37 \mathrm{pmol} / \mathrm{L}$ (eletroquimioluminescência RocheDiagnostics, Mannheim, Germany), testosterona total $>48 \mathrm{ng} / \mathrm{dL}$ (eletroquimioluminescência RocheDiagnostics, Mannheim, Germany), ou índice de andrógenos livres (IAL) $>5$ - calculado pela fórmula: testosterona total $(\mathrm{ng} / \mathrm{dL}) \times 0,0347$ / SHBG (eletroquimioluminométrico $\mathrm{MO}, \mathrm{nmol} / \mathrm{L}$ ) $\times 100$. Tanto androstenediona (quimioimunoensaio Siemens, Malvern, PA, $\mathrm{ng} / \mathrm{mL}$ ) como deidroepiandrosterona sulfato (DHEA-S) (eletroquimioluminescência, RocheDiagnostics, Mannheim, Germany, $\mathrm{ng} / \mathrm{mL}$ ) foram dosados e descritos na forma de variáveis contínuas. 


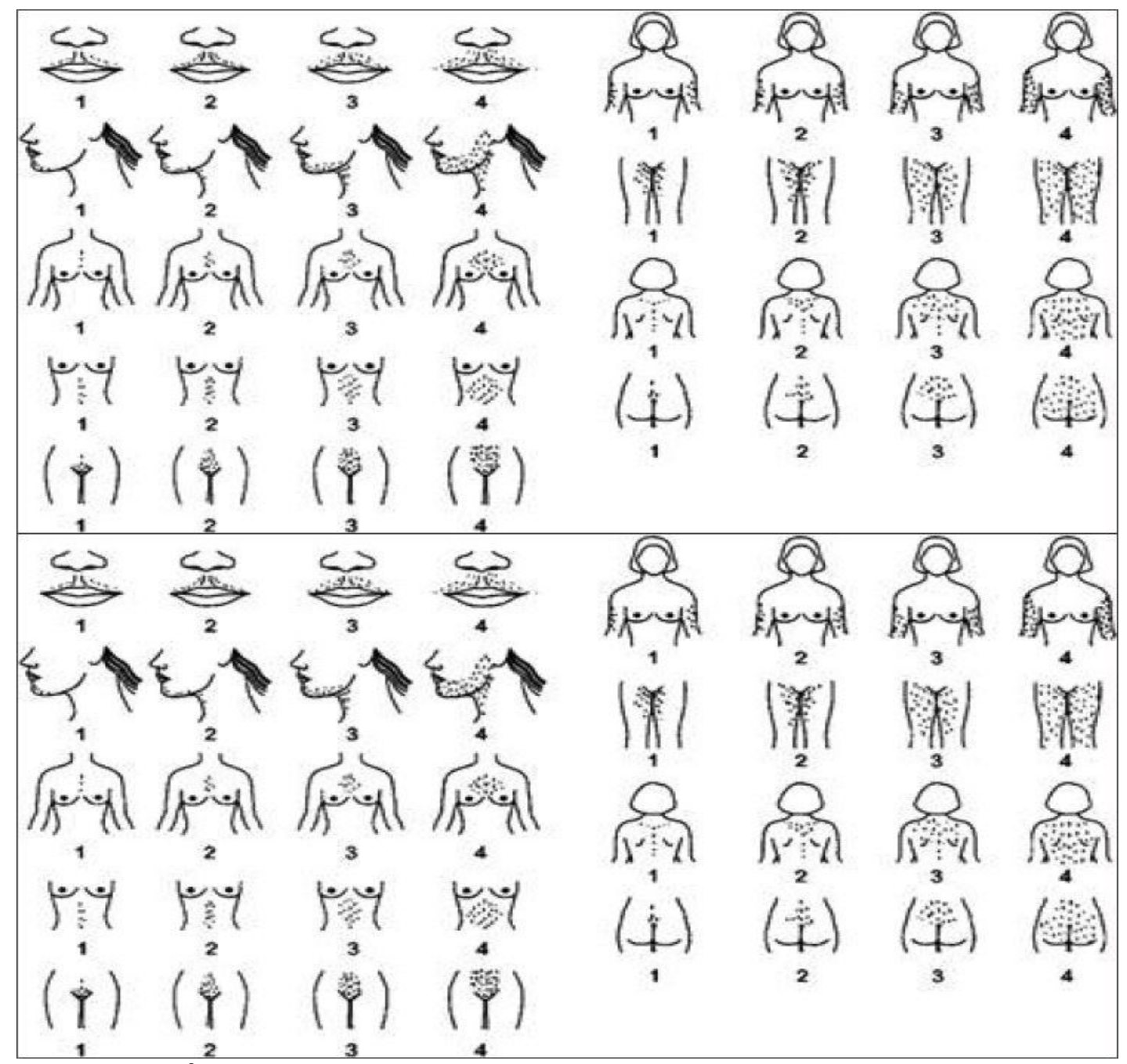

Figura 4. Índice semiquantitativo de Ferriman e Gallwey modificado para avaliação de hirsutismo ${ }^{(8)}$

\subsection{Anovulação crônica}

Para a avaliação da anovulação crônica e/ou irregularidade menstrual foi considerada a paciente com ciclo menstrual irregular nos últimos 6 meses. As dosagens de $\mathrm{LH}$ e FSH (eletroquimioluminométrico RocheDiagnostics, Mannheim, Germany, IU/L) foram realizadas, e a relação $\mathrm{LH} / \mathrm{FSH}$ foi calculada e apresentada na forma de variável contínua e categórica quando esta foi maior do que dois. 


\subsection{Ultrassonografia Ovariana}

A ultrassonografia (USG) pélvica via suprapúbica foi realizada no Setor de Radiologia do Instituto da Criança, a maioria pelo mesmo radiologista, especialista em ultrassonografia pediátrica, com o equipamento modelo Logic E9 (General Electric Medical Systems; Milwaukee, EUA), utilizando-se transdutor convexo multifrequencial de 2,8 a $5 \mathrm{MHz}$, ou com o equipamento modelo MyLab 70XVG (Esaote; Gênova, Itália), utilizando-se transdutor convexo multifrequencial de 1 a $8 \mathrm{MHz}$.

O cálculo do volume do útero e dos ovários foi obtido aplicando-se a fórmula da elipse: $L \times A P \times T \times 0,52$ ( $L=$ diâmetro longitudinal; $A P=$ diâmetro anteroposterior; $\mathrm{T}=$ diâmetro transversal; 0,52=constante da fórmula da elipse).

A qualidade da USG pélvica via suprapúbica depende, como todos os exames de USG, primeiramente do examinador, além de fatores específicos desse exame, como o preparo adequado (repleção vesical satisfatória), e do equipamento utilizado. A resolução da imagem depende da adequação da frequência do transdutor à profundidade da estrutura que se deseja estudar, sabendo-se que, quanto mais baixa a frequência, maior a profundidade atingida pela onda sonora. Nesse sentido, os transdutores multifrequenciais possibilitam a obtenção de imagens de boa resolução, mesmo em profundidades maiores, o que contorna as dificuldades encontradas na avaliação de indivíduos obesos, por exemplo.

A USG pélvica suprapúbica contudo, em geral, não tem resolução adequada para avaliar de forma fidedigna pequenas estruturas, tais como pequenos folículos ovarianos. Os maiores folículos podem ser visualizados e mensurados, porém, os pequenos folículos podem deixar de ser individualizados, sendo este o motivo pelo qual nos laudos dos exames não consta o registro do número de folículos presentes nos ovários mas o seu volume.

O volume ovariano cuja sensibilidade é de $81 \%$ e especificidade de $84 \%$ em distinguir pacientes com e sem SOP, foi escolhido como parâmetro para fazer o diagnóstico de SOP. ${ }^{(59)}$ O valor do volume ovariano maior que $10 \mathrm{~mL}$ foi escolhido como limite superior para diagnóstico de SOP, segundo todos os 
critérios, ${ }^{(35)}$ exceto a Diretriz-SEP, em que foi considerado como risco para SOP o volume ovariano maior que $12 \mathrm{~mL} .{ }^{(50)} \mathrm{A}$ ultrassonografia foi realizada de forma aleatória com relação ao ciclo menstrual das adolescentes, visto que a maioria delas apresenta ciclos muito irregulares, o que dificulta a caracterização da fase do ciclo em que se encontram no momento do exame.

\subsection{Avaliação Metabólica}

Laboratorialmente, foram avaliados os resultados do teste oral de tolerância à glicose (TOTG), com administração de $75 \mathrm{~g}$ de glicose via oral e dosagem de glicemia (enzimático colorimétrico, $\mathrm{mg} / \mathrm{dL}$ ) e de insulinemia (eletroquimioluminescência, $\mu \mathrm{U} / \mathrm{mL}$ ) nos tempos (T) zero (Glicemia de Jejum GJ), 30, 60, 90 e 120 minutos depois da ingestão da glicose. O TOTG foi realizado em todas as pacientes, pois foi previamente demonstrado ser 0 melhor método de avaliação do status glicêmico das pacientes. ${ }^{(20,60)} \quad$ Foi dosada a hemoglobina glicada (HbA1C) (HPLC - Cromatografia líquida de alta performance por troca iônica - Variant II Turbo), método certificado pelo National Glycohemoglobin Standardization Program (NGSP).

A RI foi analisada pelo cálculo do HOMA-IR [glicemia $(\mathrm{mg} / \mathrm{dL}$ ] $\mathrm{x}$ insulinemia $(\mathrm{mUl} / \mathrm{mL})$ / 405] e da relação glicemia/insulinemia de jejum.

As seguintes classificações foram adotadas: RI (HOMA-IR $\geq 2,5$ ou Relação $\mathrm{G} / \mathrm{l}<7$ ); Intolerância Oral à Glicose (IOG) (GJ entre $100-125 \mathrm{mg} / \mathrm{dL}$ ou TOTG T120 min entre $140-199 \mathrm{mg} / \mathrm{dL}$ e DM-2 (GJ $\geq 126 \mathrm{mg} / \mathrm{dL}$ ou TOTG T120 $\min \geq 200 \mathrm{mg} / \mathrm{dL}$ ou $\mathrm{HbA} 1 \mathrm{C} \geq 6,5 \%$ ).

Foi realizada a dosagem de colesterol total e frações (enzimático colorimétrico, $\mathrm{mg} / \mathrm{dL}$ ) e triglicérides (enzimático colorimétrico, $\mathrm{mg} / \mathrm{dL}$ ) e considerada dislipidemia quando $C T>200, \quad L D L>130, \quad H D L<40$ ou $\mathrm{TG}>130 \mathrm{mg} / \mathrm{dL}$.

A medida da pressão arterial (PA), foi realizada com manguito adequado para a circunferência braquial, em posição supina, por três vezes, com intervalo de pelo menos cinco minutos entre as aferições, estabelecendo-se a média das três medidas como a pressão arterial definitiva. O diagnóstico de hipertensão 
arterial sistêmica (HAS) foi feito para PA acima do percentil 95 para idade, sexo e estatura. ${ }^{(61)}$

A medida da circunferência abdominal (CA) foi feita por meio de fita centimétrica na maior circunferência abdominal, passando pela cicatriz umbilical, e avaliada de acordo com as curvas de percentil. ${ }^{(62)}$ Foi feita a relação da circunferência abdominal sobre a altura (CA/A) das pacientes. Foram considerados valores acima de 0,5 como risco de SM. ${ }^{(63)}$ A SM foi considerada quando presentes $C A>$ p95 associada a pelo menos dois dos seguintes parâmetros: $\mathrm{HDL}<40 \mathrm{mg} / \mathrm{dL} ; \mathrm{TG}>130 \mathrm{mg} / \mathrm{dL}$; HAS; glicemia de jejum $>100 \mathrm{mg} / \mathrm{dL} .{ }^{(44)}$

\subsection{Análise Estatística}

$\mathrm{O}$ z-score do IMC por idade e sexo foi calculado pela macro presente no site da Organização Mundial da Saúde. ${ }^{(64)}$

As variáveis contínuas foram descritas pelas estatísticas de posição (média, mínimo e máximo) e escala (desvio-padrão). Fizemos o teste de normalidade de Shapiro Wilk o qual mostrou que os resultados obedeciam uma curva normal. As prevalências dos fatores de risco, muitas vezes definido por cortes em variáveis contínuas, são apresentadas como frequências absolutas e relativas. As prevalências de SOP são apresentadas com os respectivas intervalos de confiança de 95\%. Para comparar as variáveis categóricas entre pacientes com e sem SOP, utilizou-se o teste exato de Fisher. E, para comparar as escalas das variáveis contínuas, utilizou-se 0 teste não paramétrico de Mann-Whitney. ${ }^{(65)}$ A análise foi realizada com auxílio do software R 3.2.0. ${ }^{(66)}$ O nível de significância dos testes foi de $5 \%$. 
4 RESULTADOS 
Todas as pacientes apresentaram IMC $\geq Z$-score +1 , sendo $5(10,2 \%)$ classificadas com sobrepeso, 26 (53,1\%) obesas e 18 (36,7\%) obesas graves.

Das 49 adolescentes avaliadas, 22 (44,9\%) eram brancas, 19 (38,8\%) pardas, $7(14,3 \%)$ negras e apenas 1 amarela. O estádio do Tanner era, em sua maioria, M5P5 e M5P6, 45 (91,8\%), com 3 casos de M4P4 (6,2\%) e 1 M5P4.

Tabela 3 - Estatísticas descritivas das variáveis contínuas. IMC: Índice de Massa Corpórea; CA: circunferência abdominal; CA/A: relação Circunferência abdominal/altura; PAS: pressão arterial sistólica; PAD: pressão arterial diastólica; LH: Hormônio Luteinizante; FSH: Hormônio Folículo Estimulante; Testo Total: Testosterona Total; Testo Livre: Testosterona Livre; IAL: Índice de Andrógenos Livres; DHEA-S: Desidroepiandrosterona Sulfato; CT: Colesterol Total; HDL: Lipoproteína de Alta Densidade; LDL: Lipoproteína de Baixa Densidade; TG: Triglicérides; HbA1C: Hemoglobina Glicada; TOTG T120: Teste De Tolerãncia Oral À Glicose, Tempo 120 Minutos; HOMA-IR: Modelo de Avaliação Homeostático da Insulina; rel G/I: Relação Glicemia/Insulinemia de Jejum

Variável

N Mínimo Máximo Média $\begin{aligned} & \text { Desvio- } \\ & \text { padrão }\end{aligned}$

$\begin{array}{llllll}\text { Idade (anos) } & 49 & 11.3 & 18,9 & 15.6 & 1.8\end{array}$

Peso (kg)

$49 \quad 58,8 \quad 133,0 \quad 91,4 \quad 18,7$

IMC $\left(\mathbf{k g} / \mathrm{m}^{2}\right)$

$49 \quad 25,0 \quad 48,7 \quad 34,6 \quad 6,3$

$\mathrm{CA}(\mathrm{cm})$

$49 \quad 80,0 \quad 146,0 \quad 109,1 \quad 14,5$

CA/A

$49 \quad 0,5$

0,9

0,7

0,1

PAS ( $\mathrm{mmHg}$ )

$49 \quad 90,0 \quad 153,3 \quad 111,7 \quad 15,5$




\begin{tabular}{|c|c|c|c|c|c|}
\hline PAD (mmHg) & 49 & 53,3 & 93,3 & 70,6 & 9,9 \\
\hline Peso ao nascer (g) & 46 & 1350,0 & 4350,0 & 3219,1 & 701,9 \\
\hline $\begin{array}{l}\text { Comprimento ao } \\
\text { nascer }(\mathrm{cm})\end{array}$ & 27 & 42,0 & 55,0 & 48,9 & 2,4 \\
\hline LH (IU/L) & 49 & 0,1 & 39,3 & 7,3 & 7,5 \\
\hline FSH (IU/L) & 49 & 0,6 & 11,7 & 4,4 & 2,1 \\
\hline LH/FSH & 49 & 0,2 & 6,1 & 1,6 & 1,3 \\
\hline Testo total (ng/dL) & 49 & 12,0 & 81,0 & 33,6 & 17,9 \\
\hline Testo livre (pmol/L) & 49 & 0,5 & 79,0 & 20,6 & 16,8 \\
\hline IAL & 49 & 0,2 & 33,7 & 4,4 & 5,2 \\
\hline DHEAS (ng/mL) & 49 & 154,0 & 4600,0 & 1801,8 & 991,2 \\
\hline $\begin{array}{l}\text { Androstenediona } \\
(\mathrm{ng} / \mathrm{mL})\end{array}$ & 49 & 0,4 & 4,5 & 1,5 & 1,0 \\
\hline $\begin{array}{l}\text { Volume ovário } \\
\text { esquerdo }(\mathrm{mL})\end{array}$ & 49 & 2,4 & 21,6 & 7,0 & 4,3 \\
\hline $\begin{array}{l}\text { Volume ovário } \\
\text { direito }(\mathrm{mL})\end{array}$ & 49 & 1,6 & 26,9 & 7,2 & 5,0 \\
\hline CT (mg/dL) & 49 & 90,0 & 237,0 & 153,1 & 29,4 \\
\hline HDL (mg/dL) & 49 & 24,0 & 75,0 & 45,3 & 11,9 \\
\hline LDL (mg/dL) & 49 & 32,0 & 178,0 & 95,9 & 273 \\
\hline
\end{tabular}




\begin{tabular}{|c|c|c|c|c|c|}
\hline $\mathrm{TG}(\mathrm{mg} / \mathrm{dL})$ & 49 & 37,0 & 205,0 & 89,9 & 43,8 \\
\hline HbA1C (\%) & 49 & 4,6 & 6,8 & 5,3 & 0,4 \\
\hline Glicemia jejum (mg/dL) & 49 & 71,0 & 99,0 & 83,1 & 6,3 \\
\hline $\begin{array}{l}\text { Insulinemia jejum } \\
(\mu \mathrm{U} / \mathrm{mL})\end{array}$ & 49 & 6,1 & 101,5 & 26,8 & 17,8 \\
\hline TOTG T120 (mg/dL) & 48 & 64,1 & 192,0 & 107,9 & 23,8 \\
\hline HOMA-IR & 49 & 1,2 & 24,8 & 5,7 & 4,3 \\
\hline 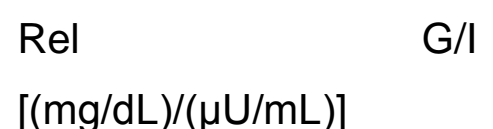 & 49 & 1,0 & 13,0 & 4,3 & 2,6 \\
\hline
\end{tabular}

As prevalências das variáveis utilizadas nos diferentes critérios diagnósticos para diagnóstico da SOP na população total do estudo são apresentadas na Tabela 4.

Tabela 4 - Prevalências das variáveis utilizadas para diagnosticar síndrome dos ovários policísticos. n: número de pacientes com a alteração; N: número total de pacientes; F-G: índice semiquantitativo de Ferriman e Gallwey; IAL: Índice de Andrógenos Livres.

\begin{tabular}{lccc}
\hline Categoria & Variáveis & IC \\
& n / N (\%) & (95\%) \\
\hline Anovulação Crônica & Ciclo menstrual irregular & $32 / 49$ & {$[50,3 ;$} \\
Hiperandrogenismo & & $(65,3 \%)$ & $77,9]$ \\
Clínico & Hirsutismo (F-G $\geq 8)$ & $8 / 49$ & {$[7,8 ;$} \\
Hiperandrogenismo & & $(16,3 \%)$ & $30,2]$ \\
Laboratorial & Testosterona total > 48 ng/dL & $9 / 49$ & {$[9,2 ;$} \\
& & $(18,4 \%)$ & $32,5]$
\end{tabular}


Testosterona livre $>37 \mathrm{pmol} / \mathrm{L}$

$5 / 49 \quad[3,8$

$(10,2 \%) \quad 23]$

$\mathrm{IAL}>5$

$14 / 49 \quad[17$

$(28,6 \%) \quad 43,5]$

Ultrassonografia

Ovariana

Volume de um dos ovários $>10 \mathrm{~mL}$

$9 / 49 \quad[10,1 ;$

$(18,4 \%) \quad 35,1]$

Volume de um dos ovários $>12 \mathrm{~mL}$

$5 / 49 \quad[4,2 ;$

$(10,2 \%) \quad 24,8]$

Tentou-se estabelecer uma relação entre o hiperandrogenismo clínico e o hiperandrogenismo laboratorial. Encontrou-se relação do hirsutismo com as concentrações de testosterona livre (Tabela 5).

Tabela 5 - Análise da relação do hiperandrogenismo clínico aferido por meio do hirsutismo pelo índice de Ferriman e Gallwey $\geq 8$, com o hiperandrogenismo laboratorial. Testo Total: Testosterona Total; Testo Livre: Testosterona Livre; IAL: Índice de Andrógenos Livres. Teste Exato de Fisher.

\begin{tabular}{|c|c|c|c|c|}
\hline & \multicolumn{3}{|c|}{ Hirsutismo (F-G $\geq 8$ ) } & \multirow[b]{2}{*}{$\mathrm{p}$} \\
\hline & Não $(n=41)$ & $\operatorname{Sim}(n=8)$ & Total $(n=49)$ & \\
\hline Testo total $>48 \mathrm{ng} / \mathrm{dL}$ & $7 / 41(17,1 \%)$ & $2 / 8(25 \%)$ & $9 / 49(18,4 \%)$ & 0,97 \\
\hline Testo livre $>37 \mathrm{pmol} / \mathrm{L}$ & $2 / 41(4,9 \%)$ & $3 / 8(37,5 \%)$ & $5 / 49(10,2 \%)$ & 0,03 \\
\hline $\begin{array}{l}\text { Androstenediona }>2,2 \\
\mathrm{ng} / \mathrm{mL}\end{array}$ & $7 / 41(17,1 \%)$ & $4 / 8(50 \%)$ & $\begin{array}{l}11 / 49 \\
(22,4 \%)\end{array}$ & 0,11 \\
\hline $\mathrm{IAL}>5$ & $10 / 41(24,4 \%)$ & $4 / 8(50 \%)$ & $14 / 4(28,6 \%)$ & 0,29 \\
\hline
\end{tabular}

Baseado nessas variáveis, calculou-se, então, o diagnóstico de SOP segundo seis critérios distintos (Tabela 6). De acordo com os critérios estabelecidos pela Diretriz-SEP, 9 meninas foram consideradas portadoras de $\mathrm{SOP}$, e foram também classificadas como SOP pelos critérios de Rotterdan, AES e NIH. 
Tabela 6 - Prevalências de síndrome dos ovários policísticos segundo critérios da Diretriz-SEP: Diretriz de Síndrome dos Ovários Policísticos na Adolescência segundo a Sociedade de Endocrinologia Pediátrica americana; NIH: Instituto Nacional de Saúde dos Estados Unidos da América, AES: Sociedade de Excesso Androgênico e Síndrome dos Ovários Policísticos; SP: Sultan \& Paris e Carmina et al.; n: número de pacientes com a alteração; N: número total de pacientes.

\begin{tabular}{lcc} 
Critério & $\mathrm{n} / \mathrm{N}(\%)$ & $\begin{array}{c}\text { IC para } \\
\text { prevalência } \\
(95 \%)\end{array}$ \\
\hline Diretriz-SEP & $9 / 49(18,4 \%)$ & {$[9,2 ; 32,5]$} \\
NIH & $10 / 49(20,4 \%)$ & {$[10,7 ; 34,8]$} \\
Rotterdam & $13 / 49(26,5 \%)$ & {$[15,4 ; 41,3]$} \\
AES & $11 / 49(22,4 \%)$ & {$[12,2 ; 37,0]$} \\
Carmina et al. & $2 / 49(4,1 \%)$ & {$[0,7 ; 15,1]$} \\
S\&P & $5 / 49(10,2 \%)$ & {$[3,8 ; 23,0]$} \\
\hline
\end{tabular}

As análises dos parâmetros clínicos e metabólicos comparando adolescentes obesas com e sem SOP definida segundo os critérios da DiretrizSEP são apresentadas na Tabela 7.

Tabela 7 - Comparação das variáveis contínuas segundo a Síndrome dos Ovários Policísticos (SOP) pelo critério da Diretriz de SOP na Adolescência segundo a Sociedade de Endocrinologia Pediátrica americana. Dados apresentados por média \pm desvio-padrão ou $\mathrm{n} / \mathrm{N}(\%)$. Teste de Mann-Whitney ou exato de Fisher. AOcomSOP: Grupo de Adolescentes Obesas com SOP; AOsemSOP: Grupo de Adolescentes Obesas sem SOP; IMC: Índice de Massa Corpórea; CA: Circunferência Abdominal; CA/A: Relação Cintura/Altura; PAS: Pressão Arterial Sistólica Média; PAD: Pressão Arterial Diastólica Média; LH: Hormônio Luteinizante; FSH: Hormônio Folículo Estimulante; IAL: Índice de Andrógenos Livres; SHBG: Proteína Ligadora dos Hormônios Sexuais; DHEAS: Desidroepiandrosterona Sulfato; CT: Colesterol Total; HDL: Lipoproteína de 
Alta Densidade; LDL: Lipoproteína de Baixa Densidade; TG: Triglicérides; HOMA-IR: Modelo de Avaliação Homeostático da Insulina; Rel G/I: Relação Glicemia/Insulinemia de Jejum; CA>p95: Circunferência Abdominal maior do que o percentil 95; HAS: Hipertensão Arterial Sistêmica; SM: Síndrome Metabólica; PIG: Pequeno para Idade Gestacional; IAL: Índice de Andrógenos Livres.

\section{Média \pm desvio-padrão}

Variável

AOcomsoP p

AOsemSOP $(n=40) \quad(n=9)$

\begin{tabular}{|c|c|c|c|}
\hline Idade (anos) & $15,4 \pm 1,8(n=40)$ & $16.3 \pm 1,9(n=9)$ & 0,167 \\
\hline IMC $\left(\mathrm{kg} / \mathrm{m}^{2}\right)$ & $34,4 \pm 6,5(n=40)$ & $35,5 \pm 5,6(n=9)$ & 0,45 \\
\hline $\mathrm{CA}(\mathrm{cm})$ & $108 \pm 15,1(n=40)$ & $113,8 \pm 11,2(n=9)$ & 0,22 \\
\hline $\mathrm{CA} / \mathrm{A}$ & $0,7 \pm 0,1(n=40)$ & $0,7 \pm 0,1(n=9)$ & 0,41 \\
\hline PAS $(\mathrm{mmHg})$ & $112,2 \pm 15,9(n=40)$ & $109,1 \pm 14,4(n=9)$ & 0,60 \\
\hline PAD $(\mathrm{mmHg})$ & $70,5 \pm 9,9(n=40)$ & $71,1 \pm 10,5(n=9)$ & 0,93 \\
\hline Peso ao nascer (g) & $\begin{array}{c}3153,2 \pm 692 \\
(n=37)\end{array}$ & $3490 \pm 717(n=9)$ & 0,20 \\
\hline Comprimento ao nascer $(\mathrm{cm})$ & $48,8 \pm 2,6(n=21)$ & $49,5 \pm 1,4(n=6)$ & 0,30 \\
\hline $\mathrm{LH}(\mathrm{IU} / \mathrm{L})$ & $7,3 \pm 8,1(n=40)$ & $7,7 \pm 4,7(n=9)$ & 0,29 \\
\hline FSH (IU/L) & $4,4 \pm 2,2(n=40)$ & $4,5 \pm 1,6(n=9)$ & 0,84 \\
\hline $\mathrm{LH} / \mathrm{FSH}$ & $1,6 \pm 1,3(n=40)$ & $1,7 \pm 0,9(n=9)$ & 0,36 \\
\hline Testosterona total (ng/dL) & $30 \pm 14,7(n=40)$ & $49,2 \pm 22,9(n=9)$ & 0,01 \\
\hline Testosterona livre (pmol/L) & $18,1 \pm 15,4(n=40)$ & $31,4 \pm 19,6(n=9)$ & 0,04 \\
\hline IAL & $4 \pm 5,4(n=40)$ & $6,3 \pm 4,2(n=9)$ & 0,04 \\
\hline Androstenediona (ng/mL) & $1,5 \pm 1(n=40)$ & $1,8 \pm 0,9(n=9)$ & 0,18 \\
\hline
\end{tabular}


DHEAS (ng/mL)

$1892,8 \pm 892,1 \quad 1397,6 \pm 1338$

$$
(n=40)
$$

$(n=9)$

0,056

Volume do ovário esquerdo $(\mathrm{mL})$

$$
6,5 \pm 4,1(n=40) \quad 9,2 \pm 4,8(n=9) \quad 0,085
$$

Volume do ovário

Direito ( $\mathrm{mL})$

\begin{tabular}{|c|c|c|c|}
\hline CT $(\mathrm{mg} / \mathrm{dL})$ & $148 \pm 25,6(n=40)$ & $175,8 \pm 35,9(n=9)$ & 0,03 \\
\hline $\mathrm{HDL}(\mathrm{mg} / \mathrm{dL})$ & $45,8 \pm 11,6(n=40)$ & $42,9 \pm 14(n=9)$ & 0,38 \\
\hline LDL (mg/dL) & $90,9 \pm 23,8(n=40)$ & $118,1 \pm 32,2(n=9)$ & 0,02 \\
\hline TG (mg/dL) & $82,7 \pm 39,7(n=40)$ & $121,7 \pm 49,3(n=9)$ & 0,015 \\
\hline HbA1C (\%) & $5,2 \pm 0,3(n=40)$ & $5,7 \pm 0,6(n=9)$ & 0,017 \\
\hline Glicemia jejum (mg/dL) & $83,2 \pm 6,4(n=40)$ & $83 \pm 5,8(n=9)$ & 0,907 \\
\hline Insulinemia jejum ( $\mu \mathrm{U} / \mathrm{mL})$ & $26,2 \pm 19,1(n=40)$ & $29,5 \pm 11(n=9)$ & 0,171 \\
\hline TOTG T120 (mg/dL) & $104,9 \pm 23,5(n=39)$ & $120,7 \pm 21,7(n=9)$ & 0,052 \\
\hline HOMA-IR & $5,6 \pm 4,6(n=40)$ & $6,1 \pm 2,6(n=9)$ & 0,187 \\
\hline Rel G/I [(mg/dL $) /(\mu \mathrm{U} / \mathrm{mL})]$ & $4,6 \pm 2,8(n=40)$ & $3,1 \pm 1(n=9)$ & 0,113 \\
\hline ETNIA (branca) & $20 / 40(50 \%)$ & $2 / 9(22,2 \%)$ & 0,23 \\
\hline ETNIA (parda) & $15 / 40(37,5 \%)$ & $4 / 9(44,4 \%)$ & \\
\hline ETNIA (negra) & $4 / 40(10 \%)$ & $3 / 9(33,3 \%)$ & \\
\hline ETNIA (amarela) & $1 / 40(2,5 \%)$ & $0 / 9(0 \%)$ & \\
\hline Sobrepeso & $5 / 40(12,5 \%)$ & $0 / 9(0 \%)$ & 0,66 \\
\hline Obesidade & $20 / 40(50 \%)$ & $6 / 9(66,7 \%)$ & \\
\hline Obesidade grave & $15 / 40(37,5 \%)$ & $3 / 9(33,3 \%)$ & \\
\hline
\end{tabular}

$6,4 \pm 4,3(n=40) \quad 10,7 \pm 6,4(n=9) \quad 0,019$ 


\begin{tabular}{|c|c|c|c|}
\hline $\mathrm{RI}$ & $34 / 40(85 \%)$ & $9 / 9(100 \%)$ & 0,577 \\
\hline $\mathrm{CA}>\mathrm{p95}$ & $37 / 40(92,5 \%)$ & $6 / 9(66,7 \%)$ & 0,067 \\
\hline $\mathrm{CA} / \mathrm{A}>0,5$ & $39 / 40(97,5 \%)$ & $9 / 9(100 \%)$ & 1 \\
\hline HAS & $12 / 40(30 \%)$ & $3 / 9(33,3 \%)$ & 1 \\
\hline $\mathrm{HDL}<40(\mathrm{mg} / \mathrm{dL})$ & $10 / 40(25 \%)$ & $4 / 9(44,4 \%)$ & 0,254 \\
\hline $\mathrm{TG} \geq 130(\mathrm{mg} / \mathrm{dL})$ & $4 / 40(10 \%)$ & $4 / 9(44,4 \%)$ & 0,028 \\
\hline \multicolumn{4}{|l|}{ Glicemia de Jejum > 100} \\
\hline$(\mathrm{mg} / \mathrm{dL})$ & $0 / 40(0 \%)$ & $0 / 9(0 \%)$ & 1 \\
\hline SM & $4 / 40(10 \%)$ & $4 / 9(44,4 \%)$ & 0,028 \\
\hline Histórico familiar + & $33 / 39(84,6 \%)$ & $5 / 8(62,5 \%)$ & 0,167 \\
\hline$P I G$ & $6 / 23(26,1 \%)$ & $1 / 6(16,7 \%)$ & 1 \\
\hline Ciclo menstrual irregular & $8 / 40(20 \%)$ & 9/9 (100\%) & 0,001 \\
\hline LH/FSH $>2$ & $10 / 40(25 \%)$ & $2 / 9(22,2 \%)$ & 1 \\
\hline Hirsutismo (F-G>8) & $3 / 40(7,5 \%)$ & $5 / 9(55,6 \%)$ & 0,003 \\
\hline Acne & $9 / 40(22,5 \%)$ & $4 / 9(44,4 \%)$ & 0,22 \\
\hline Alopecia androgênica & $7 / 40(17,5 \%)$ & $3 / 9(33,3 \%)$ & 0,364 \\
\hline \multicolumn{4}{|l|}{ Testosterona total > 48} \\
\hline $\mathrm{ng} / \mathrm{dL}$ & $4 / 40(10 \%)$ & $5 / 9(55,6 \%)$ & 0,006 \\
\hline \multicolumn{4}{|l|}{ Testosterona livre $>37$} \\
\hline $\mathrm{pmol} / \mathrm{L}$ & $2 / 40(5 \%)$ & $3 / 9(33,3 \%)$ & 0,037 \\
\hline $\mathrm{IAL}>5$ & $9 / 40(22,5 \%)$ & $5 / 9(55,6 \%)$ & 0,096 \\
\hline Volume ovariano $>10 \mathrm{~mL}$ & $6 / 40(15 \%)$ & $3 / 9(33,3 \%)$ & 0,354 \\
\hline Volume ovariano > $12 \mathrm{~mL}$ & $4 / 40(10 \%)$ & $1 / 9(11,1 \%)$ & 1 \\
\hline
\end{tabular}


A Tabela 8, enfim, compara o uso de anticoncepcional e metformina segundo o diagnóstico de SOP. Pelo teste exato de Fisher, essa amostra permite afirmar que a proporção do uso dessas medicações é superior em quem apresenta SOP diagnosticada pelo critério da Diretriz-SEP, apresentando significância estatística no caso do anticoncepcional oral.

Tabela 8 - Proporção de uso de anticoncepcional e metformina segundo a Síndrome dos Ovários Policísticos (SOP), estabelecida pelo critério da DiretrizSEP: diretriz de SOP na adolescência da Soceidade de Endocrinologia Pediátrica americana. AOcomSOP: Grupo de Adolescentes Obesas com SOP; AOsemSOP: Grupo de Adolescentes Obesas sem SOP. ${ }^{1}$ Teste exato Fisher

Variável

$\begin{array}{ccc}\underset{(n=40)}{A O s e m S O P} & \text { AOcomsOP } & \text { Total } \\ (\mathrm{n}=9) & (\mathrm{n}=49)\end{array}$

$p^{1}$

$\begin{array}{llll}\mathrm{N} & \% & \% & \%\end{array}$

\begin{tabular}{lccccccc}
\hline Anticoncepcional & $\mathbf{3}$ & $\mathbf{7 , 5}$ & $\mathbf{4}$ & $\mathbf{4 4 , 4}$ & $\mathbf{7}$ & $\mathbf{1 4 , 3}$ & $\mathbf{0 , 0 1 6}$ \\
Metformina & 24 & 60 & 7 & 77,8 & 31 & 63,3 & 0,454
\end{tabular}

Tentou-se estabelecer uma associação entre a presença dos critérios diagnósticos de SOP e SM. Poucas adolescentes apresentaram SM, de qualquer forma, ao nível de significância de 5\%, a amostra associa apenas 0 corte no índice de andrógenos livres à SM (Tabela 9)

Tabela 9 - Distribuição dos critérios de diagnóstico de Síndrome dos Ovários Policísticos segundo a presença de Síndrome Metabólica. IAL: Índice de Andrógenos Livres; AOcomSM: Grupo de Adolescentes Obesas com Síndrome Metabólica; F-G: Índice de Ferriman-Gallwey; AOsemSM: Grupo de Adolescentes Obesas sem Síndrome Metabólica.

Fator

\begin{tabular}{|c|c|c|}
\hline $\begin{array}{c}\text { AOsemSM } \\
(n=41)\end{array}$ & $\begin{array}{c}\text { AOcomSM } \\
(n=8)\end{array}$ & $\begin{array}{l}\text { Total } \\
(n=49)\end{array}$ \\
\hline
\end{tabular}


Ciclo menstrual irregular

$17 / 49$

$12 / 41(29,3 \%) \quad 5 / 8(62,5 \%) \quad(34,7 \%) \quad 0,106$

$\mathrm{LH} / \mathrm{FSH}>2$

$12 / 49$

$11 / 41(26,8 \%) \quad 1 / 8(12,5 \%) \quad(24,5 \%) \quad 0,660$

Hirsutismo

$8 / 49$

$(F-G \geq 8)$

$5 / 41(12,2 \%) \quad 3 / 8(37,5 \%) \quad(16,3 \%) \quad 0,110$

Acne

$13 / 49$

$11 / 41(26,8 \%) \quad 2 / 8(25 \%) \quad(26,5 \%) \quad 1,000$

Alopecia androgênica

$7 / 41(17,1 \%) \quad 3 / 8(37,5 \%) \quad(20,4 \%) \quad 0,333$

Testosterona total $>48$

$9 / 49$

$\mathrm{ng} / \mathrm{dL}$

$6 / 41(14,6 \%) \quad 3 / 8(37,5 \%) \quad(18,4 \%) \quad 0,151$

Testosterona livre $>37$

$5 / 49$

$\mathrm{pmol} / \mathrm{L}$

$4 / 41(9,8 \%) \quad 1 / 8(12,5 \%) \quad(10,2 \%) \quad 1,000$

Androstenediona $>2,2$

$11 / 49$

$\mathrm{ng} / \mathrm{mL}$

$9 / 41(22 \%) \quad 2 / 8(25 \%) \quad(22,4 \%) \quad 1,000$

$14 / 49$

$\mathrm{IAL}>5$

$9 / 41(22 \%) \quad 5 / 8(62,5 \%) \quad(28,6 \%) \quad 0,033$

Volume de um dos

ovários $>10 \mathrm{~mL}$

$7 / 39(17,9 \%) \quad 2 / 6(33,3 \%) \quad 9 / 45(20 \%) \quad 0,583$

Volume de um dos

$5 / 45$

ovários $>12 \mathrm{~mL}$

$4 / 39(10,3 \%) \quad 1 / 6(16,7 \%)$

$(11,1 \%) \quad 0,529$ 
5 DISCUSSÃO 
Este trabalho mostra que a prevalência de SOP em adolescentes obesas é maior do que a demonstrada na literatura. Ao adotar o critério mais recente proposto para esta população, conhecido como Diretriz-SEP, a prevalência chega a $18,4 \%$. Se adotarmos os critérios propostos para mulheres adultas, a prevalência chega a $26,5 \%$ pelos critérios de Rotterdam, $22,4 \%$ pela AES e $20,4 \%$ pelo NIH. O critério de Sultan \& Paris, muito pouco aceito pela literatura, mostrou uma prevalência de SOP de 10,2\%, e apenas quando adotamos o rigoroso critério de Carmina et al., a SOP torna-se menos prevalente, ainda assim presente em $4,1 \%$ das adolescentes obesas.

Recente trabalho brasileiro, realizado em Viçosa-MG, verificou uma prevalência de SOP em adolescentes de 15 a 18 anos de 6,2\%. ${ }^{(67)}$ Um estudo australiano de 2010, que avaliou 232 adolescentes, encontrou prevalência de SOP de $18,5 \%$ pelo critério de Rotterdam, $5 \%$ pela AES e 3,1\% pelo $\mathrm{NIH}^{(25)}$ Um estudo americano da Califórnia mostrou uma prevalência de SOP em adolescentes de $1,14 \%$ e identificou $50 \%$ de pacientes subdiagnosticadas, apesar dos sintomas apresentados. ${ }^{(52)}$ Um estudo indiano com adolescentes de 15-19 anos encontrou prevalência de $22,6 \%$ segundo os critérios de Rotterdam e de $9,8 \%$ segundo os critérios da AES. ${ }^{(68)}$

É sabido que a SOP também ocorre em mulheres sem excesso de peso, no entanto, a obesidade é uma comorbidade muito frequente, muitas vezes precedendo o desenvolvimento da SOP. ${ }^{(69)}$ No estudo de Geier et al. foram encontradas $76 \%$ das pacientes adolescentes obesas com SOP. ${ }^{(70)}$ Ollila et al. mostraram que o ganho de peso aumentou significativamente a chance de se fazer o diagnóstico de SOP, especialmente no início da idade adulta. ${ }^{(71)} \mathrm{O}$ presente trabalho mostra que a prevalência de SOP em adolescentes obesas é maior que as encontradas em trabalhos que avaliaram adolescentes em geral, concordando com a possibilidade da obesidade poder ser um fator de risco para a SOP. Esta observação é importante, porque pode identificar um período de tempo sensível quando o ganho de peso desempenha um papel crucial nas ações de prevenção. 
Christensen et al. demonstraram que adolescentes com sobrepeso, obesidade e obesidade grave tem risco de 3,85, 10,25 e 23,1 vezes maior de desenvolver SOP, respectivamente. ${ }^{(52)}$ Apesar disso, não encontramos diferença estatística entre os graus de obesidade das adolescentes obesas com e sem SOP pela Diretiz-SEP.

Sintomas de SOP aparecem desde a puberdade e podem se confundir com o desenvolvimento puberal normal. Flutuações hormonais durante este período tornam o diagnóstico de SOP mais difícil. A heterogeneidade dos sinais clínicos e a falta de uniformidade em definir os sintomas confundem muito o diagnóstico, principalmente na população adolescente. A recente Diretriz-SEP tenta estabelecer critérios mais rigorosos para se estabelecer o diagnóstico de SOP nessa faixa etária. ${ }^{(50)}$ Ao utilizarmos este critério, ainda assim, encontramos uma alta prevalência de SOP nas adolescentes obesas (18,4\%).

Para West et al., a irregularidade menstrual se mostrou ser um bom marcador de hiperandrogenismo e foi tida como o sintoma inicial da SOP, ${ }^{(20)}$ tendo sensibilidade de $50,5 \%$ e especificidade de $73,2 \%$, valor preditivo positivo de 0,9 e negativo de 0,7 para predizer SOP aos 26 anos de idade. ${ }^{(72)}$ Entre as adolescentes obesas pesquisadas, mesmo depois de terem sido excluídas do estudo as adolescentes que tinham apresentado a menarca há menos de dois anos, 65,3\% referiam-se a irregularidade menstrual. Hickey et al. encontraram $51,7 \%$ de irregularidade menstrual entre 232 adolescentes avaliadas na Austrália. ${ }^{(25)}$ Tamanha alta prevalência de irregularidade menstrual demonstra que esta é uma alteração muito comum na adolescência e parece não ser um bom marcador de SOP em adolescentes obesas. Foi demonstrado que a irregularidade menstrual na adolescência, que persiste na idade adulta, está associada à SM, disglicemia, DM2 e SOP. ${ }^{(73,74)}$ Nosso estudo não encontrou diferença estatística com relação à irregularidade menstrual nas adolescentes obesas com e sem SM, embora as AOcomSOP apresentassem estatisticamente mais SM que as AOsemSOP.

Um estudo finlandês, com seguimento de 10 anos, demonstrou que a irregularidade menstrual e/ou a hiperandrogenemia laboratorial, medida por meio do IAL, estão alteradas em mulheres com SOP na vida adulta desde a adolescência, independente do peso e/ou ganho de peso. ${ }^{(72)}$ Yoo et al. 
demonstraram que adolescentes obesas que referiam oligomenorreia, porém sem hiperandrogenismo clínico ou laboratorial, apresentavam a secreção de gonadotrofinas muito semelhante à de adolescentes obesas com SOP, sugerindo que a avaliação laboratorial das gonadotrofinas é de suma importância para o diagnóstico precoce da síndrome. ${ }^{(75)}$ No presente estudo, a relação LH/FSH não foi estatisticamente significante entre os grupos de adolescentes obesas com e sem SOP quando adotamos os critérios da Diretriz-SEP ( $p=0.366)$.

O hirsutismo $(F-G \geq 8)$ é descrito como pouco prevalente na população adolescente, ${ }^{(25)}$ entretanto, encontramos uma prevalência relativamente alta entre adolescentes obesas (16,5\%), e este se mostrou mais prevalente nas AOcomSOP $(p<0.001)$. Avaliar a função ovariana androgênica parece ter valor prognóstico para SOP, mesmo em meninas com hiperandrogenismo funcional. ${ }^{(76)}$ Yoo et al. também demonstraram que apesar dessas adolescentes obesas apresentarem concentrações de andrógenos duas vezes menores que as adolescentes com SOP, estas já eram superiores a dos controles não obesos. ${ }^{(75)}$ Este estudo demonstrou diferença estatística com relação às dosagens de testosterona total e livre e do IAL entre AOcomSOP e AOsemSOP. Ao relacionar hirsutismo com os sinais de hiperandrogenismo laboratorial, foi encontrada relação positiva com a concentração de testosterona livre, que está de acordo com a literatura. ${ }^{(25)}$

$\mathrm{Na}$ literatura, há uma controvérsia com relação à utilidade do volume ovariano como parâmetro diagnóstico de ovários policísticos. Youngster et al. consideram que a ultrassonografia pélvica via suprapúbica pode ser útil como ferramenta diagnóstica de SOP em adolescentes, mesmo com a presença de obesidade. ${ }^{(77)}$ Ao contrário, para Fruzetti et al. a ultrassonografia pélvica via suprapúbica mostrou ter uma baixa sensibilidade para o diagnóstico de SOP em adolescentes. ${ }^{(37)} \mathrm{O}$ presente estudo demonstrou uma prevalência de 18,4\% de adolescentes obesas com volume ovariano acima de $10 \mathrm{~mL}$, próxima à reportada na literatura de $25,7 \%,{ }^{(25)}$ a qual cai para $10,2 \%$ se for adotado o valor de $12 \mathrm{~mL}$. Ao se analisar as 9 pacientes classificadas como portadoras de SOP pelo critério da Diretriz-SEP, apenas três apresentaram volume ovariano acima de $10 \mathrm{~mL}$ e uma acima de $12 \mathrm{~mL}$. 
Foi demonstrado que meninas PIG, principalmente as que recuperaram o crescimento até os dois anos de idade, apresentam maiores níveis de hiperinsulinemia, assim como maior chance de desenvolver adrenarca precoce ou exagerada e, consequentemente, maior risco de SOP. ${ }^{(42,43)}$ Dentre as adolescentes obesas com e sem SOP, segundo os critérios da Diretriz-SEP, não foi encontrada diferença estatística com relação ao peso ou comprimento ao nascer.

É aceito que a puberdade é caracterizada por um aumento da RI e que poderia induzir sintomas semelhantes aos da SOP, desaparecendo após 0 período puberal. ${ }^{(78)} \mathrm{A}$ obesidade e o consequente aumento da gordura abdominal visceral também predispõe à $\mathrm{RI}$ e SM, induzindo a anovulação crônica e desencadeando o hiperandrogenismo. ${ }^{(79)}$ Roe et al. demonstraram que a prevalência de sobrepeso e obesidade foi significativamente maior em adolescentes com SOP do que sem SOP (60\% vs 18\%). ${ }^{(80)}$ Adolescentes com SOP são mais obesas que adolescentes sem SOP e têm mais fatores de risco para $S M$, inclusive maior RI. ${ }^{(80,81)}$ Este estudo não encontrou diferença entre os graus de obesidade entre AOcomSOP e AOsemSOP $(p=0,66)$, tampouco na RI.

Um estudo com meninas obesas com e sem SOP, pareadas pela gordura visceral medida pela ressonância magnética de abdome, em que se avaliou a sensibilidade insulínica por meio de clamp hiperinsluinêmicoeuglicêmico, mostrou que as adolescentes com SOP tinham SI 50\% mais reduzida. ${ }^{(20)}$ Há uma correlação positiva entre os componentes da SM e SOP. ${ }^{(52,82-85)}$ Crianças e adolescentes com sinais de RI manifestada por meio de Acanthosis nigricans, de SM ou com história familiar positiva de SOP apresentam maior risco de desenvolver SOP. ${ }^{(6)}$ É descrito que na SOP a distribuição de gordura central é mais comum, com obesidade visceral predominando sobre gordura periférica. ${ }^{(86)}$. Apesar de Palmert et al. terem encontrado uma presença de $33 \%$ de IOT e DM-2 em adolescentes com SOP avaliadas pelo TOTG $^{(60)}$, o presente estudo não encontrou associação entre RI medida por meio do HOMA-IR ou da relação glicemia/insulina, tampouco na avaliação de IOG ou DM-2 por meio do TOTG entre as AOcomSOP e AOsemSOP, exceto ao se analisar os valores de $\mathrm{HbA1C}(p=0.017)$, o qual, 
apesar de apresentar um valor maior nas AOcomSOP, ainda assim estavam dentro dos limites da normalidade.

Não foram encontradas diferenças estatísticas nas medidas de CA entre as AOcomSOP e AOsemSOP. A relação $C / A$, descrita como bom parâmetro para avaliação de SM em crianças e adolescentes, ${ }^{(63)}$ também não foi diferente entre os grupos.

Foram localizadas diferenças entre a presença de SM em adolescentes obesas com e sem SOP ( $p=0,028)$, e a variável isolada que diferenciou os grupos foi a dosagem dos TG acima de $130 \mathrm{mg} / \mathrm{dL}$. Isso vai de acordo com a literatura, que aponta a presença de $37 \%$ de SM em adolescentes obesas com SOP versus $5 \%$ das adolescentes controles. ${ }^{(86)}$ Ao se analisar as variáveis metabólicas isoladamente de forma contínua notamos que as dosagens do CT $(p=0,033)$, do LDL $(p=0,022)$ e do TG $(p=0,017)$ apresentavam-se diferentes entre os grupos, sendo o CT considerado alto e o LDL e o TG limítrofes nas AOcomSOP. Esses achados vão de acordo com a literatura e sugerem que a SOP, em adolescentes obesas, é um fator de risco para SM. ${ }^{(15)}$

Ao se classificar todas as adolescentes do estudo como tendo ou não $\mathrm{SM}$, foi encontrado que o único fator de risco presente nos critérios diagnósticos da SOP coincidente com as adolescentes com SM é o $\mid A L>5$ $(p=0.033)$.

Este é um estudo transversal, que apresenta um pequeno tamanho amostral, sujeito às limitações típicas de estudos observacionais e ao erro tipo 2, podendo prejudicar a análise dos resultados. A ultrassonografia pélvica via suprapúbica e as dosagens hormonais foram realizadas em períodos aleatórios do ciclo menstrual das adolescentes. A variável irregularidade menstrual foi avaliada por meio do questionamento das pacientes e de seus responsáveis. Não foi possível recuperar informações sobre a pubarca e/ou a adrenarca das pacientes. O IAL leva no denominador de sua fórmula a dosagem da SHBG, a qual se encontra diminuída na obesidade. ${ }^{(25)}$

Outro possível viés deste estudo seria o uso de metformina para tratamento da RI pela maioria das adolescentes da amostra, uma vez que esta droga contribui para a perda de peso e a prevenção da progressão da RI para DM-2. ${ }^{(87)}$ A metformina é também utilizada para o tratamento da SOP. A 
hiperinsulinemia é vista como um dos fatores etiopatogênicos da doença, ${ }^{(20)}$ e já foi demostrado que o tratamento com metformina melhora o quadro de hiperandrogenismo em adolescentes. ${ }^{(88)} \mathrm{O}$ uso da metformina não mostrou diferença estatística entre as adolescentes com e sem SOP. Outro estudo não encontrou mudanças no peso de pacientes obesas com SOP que usaram metformina como parte do tratamento. ${ }^{(70)}$

O uso de ACO entre as adolescentes classificadas com e sem SOP, segundo dos critérios pospostos pela Diretriz-SEP, foi estatisticamente significante. O maior uso de ACO em AOcomSOP quando comparado à AOsemSOP foi surpreendente, pois se esperava encontrar um maior número de pacientes em uso dessas medicações no grupo sem a doença, uma vez que as medicações deveriam normalizar as alterações observadas na SOP.

Há uma controvérsia na literatura quanto a necessidade de se estabelecer o diagnóstico de SOP na adolescência, uma vez que isso poderia ter um impacto importante na qualidade de vida dessas pacientes e gerar, desnecessariamente, ansiedade com relação à futura fertilidade dessas meninas. Spritzer et al. conclui em seu trabalho que a SOP é subdiagnosticada na adolescência e que alguns sinais poderiam indicar ao médico a suspeita da síndrome, dentre eles, a obesidade. ${ }^{\left({ }^{89)}\right.}$ As diretrizes atuais recomendam tratar as manifestações individuais da SOP, isso sugere que, independente do diagnóstico da SOP, meninas estariam sendo tratadas de acordo com os sinais clínicos da SOP. ${ }^{(90)}$ Entretanto, há quem recomende que em uma situação clínica em que haja suspeita de SOP seja realizada uma investigação tanto laboratorial quanto ultrassonográfica, já que nem todos os sinais clínicos, laboratoriais e ultrassonográficos podem estar presentes na mesma paciente. $^{(40)}$ 
6 CONCLUSÕES 
Ao final desta dissertação, concluímos que:

- A prevalência de SOP em adolescentes obesas é alta quando comparada aquela observada na literatura. Ao se adotar o mais recente critério diagnóstico proposto para adolescentes (Diretriz-SEP), foi encontrada prevalência de $18,4 \%$, chegando a $26,5 \%$ quando adotados os critérios propostos para mulheres adultas (Rotterdam).

- $O$ hirsutismo, definido como índice de $F-G \geq 8$, está associado às concentrações plasmáticas de testosterona livre em adolescentes obesas.

- Adolescentes obesas com SOP pelo mais novo critério da Diretriz-SEP, quando comparadas às adolescentes obesas sem SOP, não apresentam diferença de etnia tão pouco do grau de obesidade. A relação circunferência abdominal/altura não foi diferente, assim como não encontramos maior prevalência de PIG nesse grupo. No entanto, as adolescentes obesas com SOP apresentaram maiores índices de dislipidemia (aumento de CT, LDL e $\mathrm{TG}$ ) assim como maiores dosagens de $\mathrm{HbA1C}$, mesmo que estes ainda tenham se mantido dentro da normalidade.

- Adolescentes obesas com SOP apresentam taxas de síndrome metabólica maiores que adolescentes obesas sem SOP $(p=0.028)$. Dessas, o único fator de risco presente nos critérios diagnósticos da SOP coincidente com as adolescentes com SM foi o IAL>5 ( $p=0.033)$, assim como na literatura. 
7 CONSIDERAÇÕES FINAIS 
Devido à alta prevalência de SOP em adolescentes obesas, achamos muito adequado que todas as pacientes obesas, pós menarca, sejam submetidas a uma triagem laboratorial e ultrassonográfica para SOP, o que reduziria o número de adolescentes não diagnosticadas e permitiria a eventual intervenção precoce, minimizando morbidade futura e infertilidade.

O critério mais estrito de diagnóstico (Diretriz-SEP) de SOP tenta evitar que muitas adolescentes normais sejam "rotuladas" como portadoras da síndrome, já que a puberdade normal acarreta alterações físicas, laboratoriais e de imagem muito semelhantes às da SOP. Mesmo com este critério mais rigoroso, 18,4\% dessas meninas enquadraram-se no diagnóstico. Em resumo, não devemos nos "esquecer" desse diagnóstico, especialmente quando estivermos diante de adolescentes com sobrepeso, obesas ou obesas grave. 
8 ANEXOS 


\title{
Anexo A
}

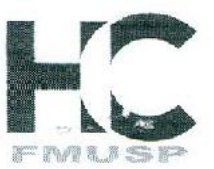

\author{
Hospital das Clínicas da FMUSP \\ 1139 \\ Comissão de Ética para Análise de Projetos de Pesquisa - CAPPesq
}

\section{PROJETO DE PESQUISA}

Título: AVALIAÇÃO DA PREVALÊNCIA E DAS CARACTERÍSTICAS DA SÍNDROME DO OVÁRIO POLICÍSTICO NAS PACIENTES DO AMBULATÓRIO DE OBESIDADE DA DISCIPLINA DE ENDOCRINOLOGIA PEDIÁTRICA DO INSTITUTO DA CRIANÇA DO HOSPITAL DAS CLÍNICAS DA FACULDADE DE MEDICINA DA USP Pesquisador Responsável: Dr. Durval Damiani

Versão: 1

Pesquisador Executante: Dra. Marina Ybarra

CAAE: 24865513.5 .0000 .0068

Co-autores: Dra. Raissa Beltrão Sampaio, Dra Ruth Rocha Franco,Dra Louise Cominato

Finalidade Acadêmica Mestrado

Orientador: Dr Durval Damiani

Instituição: HCFMUSP

Departamento: PEDIATRIA

\section{PARECER CONSUBSTANCIADO DO CEP}

Registro on-line: 11446

Número do Parecer: 513.833

Data da Relatoria: 22/01/2014

Apresentação do Projeto: A Síndrome do Ovário Policístico (SOP) é a doença endocrinológica mais frequente em mulheres em idade fértil, podendo aparecer já na primeira década de vida. Contudo, costuma surgir na adolescência quando há a maturação do eixo hipotálamo-hipófise-gonadal. Muitas vezes esta síndrome não é percebida pelo médico, pois suas alterações se confundem com as mudanças típicas da adolescência. Os pesquisadores propõem um estudo transversal no qual serão investigadas as características demográficas quanto à idade, etnia, histórico familiar, tanto durante as consultas de rotina do ambulatório como pela revisão de prontuários médicos. Serão incluídas 131 pacientes do sexo feminino, entre 10 e 18 anos de idade, matriculadas e ativas no Ambulatório de Obesidade da Unidade de Endocrinologia Pediátrica do Departamento de Pediatria do Instituto da Criança. Todas as pacientes matriculadas neste ambulatório tem diagnóstico de obesidade ou sobrepeso, definido através da medida do índice de massa corpórea (IMC) acima do percentil 85 para idade e sexo. A SOP será definida de acordo com critérios clínicos e bioquímicos. Será também realizada ultrassonografia pélvica para avaliar a presença de ovários policísticos.

Objetivo da Pesquisa: $O$ objetivo secundário é conhecer quais são as características clínicas, bioquímicas e ultrassonográficas mais frequentes da SOP nesta população específica, de crianças e adolescentes obesas do sexo feminino o objetivo secundário é conhecer quais são as características clínicas, bioquímicas e ultrassonográficas mais frequentes da SOP nesta população específica, de crianças e adolescentes obesas do sexo feminino

Avaliação dos Riscos e Benefícios: Riscos: Não há riscos diretos à saúde das pacientes, além dos riscos inerentes aos exames de sangue, muito embora ambos já são realizados de rotina no Ambulatório de Obesidade. Será mantida a confidencialidade e a integridade dos dados do prontuário. Para maior proteção e confiabilidade, os prontuários serão examinados por um grupo limitado de pesquisadores, dentro da instituição, não sendo permitida a reprodução dos documentos do prontuário. A identidade das pacientes também será preservada, sendo utilizada na análise do banco de dados apenas a sigla das iniciais dos nomes. Benefícios: Este estudo proporcionará benefícios diretos às pacientes envolvidas,

Rua Dr. Ovídio Pires de Campos, 225 - Prédio da Administração - 5o andar

CEP 05403-010 - São Paulo - SP.

5511 2661-7585 - 5511 2661-6442 ramais: 16, 17, 18 | marcia.carvalho@hc.fm.usp.br 


\section{Hospital das Clínicas da FMUSP}

Comissão de Ética para Análise de Projetos de Pesquisa - CAPPesq

pois poderão receber tanto o diagnóstico da doença como o tratamento direcionado e consequente prevenção precoce das comorbidades envolvidas nesta patologia. Além disso, os resultados deste estudos podem contribuir para o aumento do conhecimento, dentro da comunidade médica, sobre este tema.

Comentários e Consideraçães sobre a Pesquisa: Projeto bem apresentado e bem delineado, que trará contribuições para a área do conhecimento.

Consideraçōes sobre os Termos de apresentação obrigatória: Termos estão apropriados, sem pendências éticas.

Recomendaçס̃es: sem recomendações

Conclusões ou Pendências e Lista de Inadequações: Aprovado

Situação do Parecer: Aprovado

Necessita Apreciação da CONEP: Não.

Considerações Finais a critério do CEP: Em conformidade com a Resolução CNS ne 466/12 - cabe ao pesquisador: a) desenvolver o projeto conforme delineado; b) elaborar e apresentar relatórios parciais e final; c)apresentar dados solicitados pelo CEP, a qualquer momento; d) manter em arquivo sob sua guarda, par 5 anos da pesquisa, contendo fichas individuais e todos os demais dacumentos recomendados pelo CEP; e) encaminhar os resultados para publicação, com as devidos créditos aos pesquisadores associados e ao pessoal técnico participante do projeto; f) justificar perante ao CEP interrupção do projeto ou a não publicação dos resultados.

Săo Paulo, 23 de Janeiro de 2014

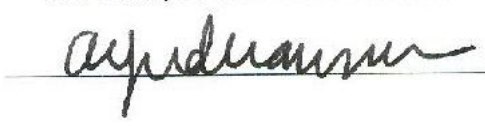

Prof. Dr. Alfredo José Mlansur
-Coordenador

Comissão ce Élica para Análise de

Projetos de Pequisa - CAPPEs

Rua Dr. Ovídio Pires de Campos, 225 - Prédio da Administraçăo - 50 andar

CEP 05403-010 - Săo Paulo - SP.

5511 2661-7585 - 5511 2661-6442 ramais: 16, 17, 18 | marcia.carvalho@hc.fm.usp.br 


\section{Anexo B}

HOSPITAL DAS CLÍNICAS DA FACULDADE DE MEDICINA DA
UNIVERSIDADE DE SÃO PAULO - HCFMUSP

TERMO DE CONSENTIMENTO LIVRE E ESCLARECIDO

DADOS DE IDENTIFICAÇÃO DO SUJEITO DA PESQUISA OU RESPONSÁVEL LEGAL

1-NOME:

DOCUMENTO DE IDENTIDADE №:

SEXO: $M \quad F$

DATA NASCIMENTO: ........................

ENDEREÇO:

BAIRRO:

TELEFONE: DDD (.....).

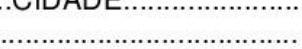

2. RESPONSÁVEL LEGAL

NATUREZA (grau de parentesco, tutor, curador etc.):

DOCUMENTO DE IDENTIDADE:

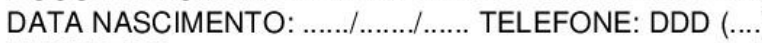

ENDEREÇO:

BAIRRO:

CIDADE

.SEXO: $M\urcorner F\ulcorner$

$\mathrm{N}^{\mathrm{o}}$ ......... APTO:

\section{DADOS SOBRE A PESQUISA}

1. TÍTULO DO PROTOCOLO DE PESQUISA: Avaliação da prevalência e das características da Síndrome dos Ovários Policísticos (SOP) em pacientes obesas PESQUISADOR: Dr. Durval Damiani

CARGO OU FUNÇÃO: Chefe da Unidade de Endocrinologia Pediátrica do ICr - HC - FMUSP CRM:

2. AVALIAÇÃO DO RISCO DA PESQUISA

RISCO MÍNIMO $\square \quad$ RISCO ALTO

RISCO BAIXO RISCO MAIOR

3. DURAÇÃO DA PESQUISA: 1 ANO 


\section{REGISTRO DAS EXPLICAÇÕES DO PESQUISADOR AO PACIENTE OU SEU REPRESENTANTE LEGAL SOBRE A PESQUISA CONSIGNANDO:}

Prezado(a) senhor(a), sua filha tem uma doença crônica grave que é a obesidade. $O$ tratamento da obesidade é feito com dietas, mudança no estilo de vida (como atividade física) e, em alguns casos, pode-se conseguir auxílio com alguns tipos de medicações.

Além das diversas complicações já conhecidas, a obesidade pode levar a alterações do colesterol e ao diabetes mellitus tipo 2. A obesidade também tem sido associada a uma doença conhecida como Síndrome do Ovário Policístico. Esta síndrome acomete meninas adolescentes e cursa, por exemplo, com alterações na menstruação, aumento de pelos e de espinhas. Na ultrassonografia, muitas vezes, se detecta a presença de cistos nos ovários.

Este estudo visa descobrir se as pacientes com excesso de peso, acompanhadas neste ambulatório, apresentam esta doença ou sinais dela. A proposta é descobrir se sua filha tem a Síndrome dos Ovários Policísticos.

As consultas serão realizadas com as consultas do Ambulatório de Obesidade. Os exames serão solicitados com os exames de rotina do Ambulatório de Obesidade.

Para a realização de exames, o sangue é colhido com seringas e agulhas usadas uma única vez (descartáveis), a partir de uma veia sob a pele. Para a realização da ultrassonografia, utiliza-se de um aparelho que desliza na barriga por meio de um gel.

No local da picada da agulha poderá ser sentido um pouco de dor e, às vezes, poderá ficar uma mancha roxa, que sumirá sozinha em poucos dias.

Se o(a) senhor(a) não quiser que sua filha participe deste estudo tem todo o direito de recusar, e ela continuará a receber o tratamento adequado para a obesidade. Caso comece neste estudo e depois deseje desistir, também estará garantida a continuidade do tratamento da sua filha da melhor forma possível.

O principal investigador é o Dr. Durval Damiani, que pode ser encontrado no Instituto da Criança do Hospital das Clínicas da Faculdade de Medicina da Universidade de São Paulo, localizado na Av. Dr. Enéas Carvalho de Aguiar, 647 - São Paulo - SP, 05403-000, Brasil Telefone: (0xx)11 3069-9832.

Se você tiver alguma consideração ou dúvida sobre a ética da pesquisa, entre em contato com o Comitê de Ética em Pesquisa (CEP) - Rua Ovídio Pires de Campos, 225 - $5^{\circ}$ andar Telefone: (0xx)11 3069-6442 ramais 16, 17, 18 ou 20. FAX: (0xx)11 3069-6442 ramal 26 - E-mail: cappesq@hcnet.usp.br

É garantida a liberdade da retirada de consentimento a qualquer momento e deixar de participar do estudo, sem qualquer prejuízo à continuidade de seu tratamento na Instituição.

As informações obtidas serão analisadas em conjunto com outros pacientes, não sendo divulgado a identificação de nenhum paciente. $O$ (a) senhor(a) tem o direito de ser mantido(a) atualizado(a) sobre os resultados parciais das pesquisas, quando em estudos abertos, ou de resultados que sejam do conhecimento dos pesquisadores.

Não há despesas pessoais para o participante em qualquer fase do estudo, incluindo exames e consultas. Também não há compensação financeira relacionada à sua participação. Se existir qualquer despesa adicional, ela será absorvida pelo orçamento da pesquisa.

Há um compromisso do pesquisador de utilizar os dados e o material coletado somente para esta pesquisa. 
Acredito ter sido suficientemente informado a respeito das informações que li ou que foram lidas para mim, descrevendo o estudo "Avaliação da Prevalência e das Características da Síndrome dos Ovários Policísticos (SOP) em adolescentes obesas".

Eu discuti com o Dr. Durval Damiani sobre a minha decisão em participar deste estudo. Ficaram claros para mim quais são os propósitos do estudo, os procedimentos a serem realizados, seus desconfortos e riscos, as garantias de confidencialidade e de esclarecimentos permanentes.

Ficou claro, também, que minha participação é isenta de despesas e que tenho garantia do acesso a tratamento hospitalar quando necessário. Concordo voluntariamente em participar deste estudo e poderei retirar o meu consentimento a qualquer momento, antes ou durante o andamento do estudo, sem penalidades, ou prejuízo, ou perda de qualquer benefício que eu possa ter adquirido, ou no meu atendimento neste Serviço.

Assinatura do paciente/representante legal

Assinatura da testemunha
Data

Data

Para casos de pacientes menores de 18 anos, analfabetos, semianalfabetos ou portadores de deficiência auditiva ou visual.

Declaro que obtive de forma apropriada e voluntária o Consentimento Livre e Esclarecido deste paciente ou representante legal para a participação neste estudo.

Assinatura do responsável pelo estudo

Data 
9 REFERÊNCIAS 
1. Stein I, Leventhal, ML. Amenorrhea associated qith bilateral polycistic ovaries. Am J Obstet Gynecol. 1935;29:181-91.

2. Orsino A, Van Eyk N, Hamilton J. Clinical features, investigations and management of adolescents with polycystic ovary syndrome. Paediatr Child Health. 2005;10(10):602-8.

3. Azziz R, Sanchez LA, Knochenhauer ES, Moran C, Lazenby J, Stephens KC, et al. Androgen excess in women: experience with over 1000 consecutive patients. The Journal of clinical endocrinology and metabolism. 2004;89(2):45362.

4. Franks S. Polycystic ovary syndrome in adolescents. International journal of obesity. 2008;32(7):1035-41.

5. Yun JH, Choi JW, Lee KJ, Shin JS, Baek KH. The promoter -1031(T/C) polymorphism in tumor necrosis factor-alpha associated with polycystic ovary syndrome. Reprod Biol Endocrinol. 2011;9:131.

6. Rosenfield RL. Clinical review: Identifying children at risk for polycystic ovary syndrome. The Journal of clinical endocrinology and metabolism. 2007;92(3):787-96.

7. Silfen ME, Denburg MR, Manibo AM, Lobo RA, Jaffe R, Ferin M, et al. Early endocrine, metabolic, and sonographic characteristics of polycystic ovary syndrome (PCOS): comparison between nonobese and obese adolescents. The Journal of clinical endocrinology and metabolism. 2003;88(10):4682-8. 
8. Ferriman D, Gallwey JD. Clinical assessment of body hair growth in women. The Journal of clinical endocrinology and metabolism. 1961;21:1440-7. 9. Azziz R, Carmina E, Sawaya ME. Idiopathic hirsutism. Endocr Rev. 2000;21(4):347-62.

10. Baillargeon JP, luorno MJ, Nestler JE. Insulin sensitizers for polycystic ovary syndrome. Clinical obstetrics and gynecology. 2003;46(2):325-40.

11. Diamanti-Kandarakis E, Dunaif A. Insulin resistance and the polycystic ovary syndrome revisited: an update on mechanisms and implications. Endocr Rev. 2012;33(6):981-1030.

12. Bach LA. The insulin-like growth factor system: basic and clinical aspects. Aust N Z J Med. 1999;29(3):355-61.

13. Dewailly D, Lujan ME, Carmina E, Cedars MI, Laven J, Norman RJ, et al. Definition and significance of polycystic ovarian morphology: a task force report from the Androgen Excess and Polycystic Ovary Syndrome Society. Human reproduction update. 2014;20(3):334-52.

14. Trent M, Austin SB, Rich M, Gordon CM. Overweight status of adolescent girls with polycystic ovary syndrome: body mass index as mediator of quality of life. Ambul Pediatr. 2005;5(2):107-11.

15. Ojaniemi M, Tapanainen P, Morin-Papunen L. Management of polycystic ovary syndrome in childhood and adolescence. Hormone research in paediatrics. 2010;74(5):372-5.

16. Elizondo-Montemayor L, Hernandez-Escobar C, Lara-Torre E, Nieblas B, Gomez-Carmona M. Gynecologic and Obstetric Consequences of Obesity in Adolescent Girls. Journal of pediatric and adolescent gynecology. 2016. 
17. Kiddy DS, Hamilton-Fairley D, Bush A, Short F, Anyaoku V, Reed MJ, et al. Improvement in endocrine and ovarian function during dietary treatment of obese women with polycystic ovary syndrome. Clinical endocrinology. 1992;36(1):105-11.

18. Nestler JE, Barlascini CO, Matt DW, Steingold KA, Plymate SR, Clore JN, et al. Suppression of serum insulin by diazoxide reduces serum testosterone levels in obese women with polycystic ovary syndrome. The Journal of clinical endocrinology and metabolism. 1989;68(6):1027-32.

19. Witchel SF, Arslanian S. Ovarian responses to hCG stimulation: insulin resistance/hyperinsulinaemia vs. insulin deficiency. Clinical endocrinology. $1999 ; 51(1): 127-30$.

20. Lewy VD, Danadian K, Witchel SF, Arslanian S. Early metabolic abnormalities in adolescent girls with polycystic ovarian syndrome. The Journal of pediatrics. $2001 ; 138(1): 38-44$.

21. Legro RS, Arslanian SA, Ehrmann DA, Hoeger KM, Murad MH, Pasquali $\mathrm{R}$, et al. Diagnosis and treatment of polycystic ovary syndrome: an Endocrine Society clinical practice guideline. J Clin Endocrinol Metab. 2013;98(12):456592.

22. Knochenhauer ES, Key TJ, Kahsar-Miller M, Waggoner W, Boots LR, Azziz R. Prevalence of the polycystic ovary syndrome in unselected black and white women of the southeastern United States: a prospective study. The Journal of clinical endocrinology and metabolism. 1998;83(9):3078-82.

23. Metcalf MG, Skidmore DS, Lowry GF, Mackenzie JA. Incidence of ovulation in the years after the menarche. The Journal of endocrinology. $1983 ; 97(2): 213-9$. 
24. Fauser BC, Tarlatzis BC, Rebar RW, Legro RS, Balen AH, Lobo R, et al. Consensus on women's health aspects of polycystic ovary syndrome (PCOS): the Amsterdam ESHRE/ASRM-Sponsored 3rd PCOS Consensus Workshop Group. Fertility and sterility. 2012;97(1):28-38 e25.

25. Hickey M, Doherty DA, Atkinson H, Sloboda DM, Franks S, Norman RJ, et al. Clinical, ultrasound and biochemical features of polycystic ovary syndrome in adolescents: implications for diagnosis. Human reproduction. 2011;26(6):1469-77.

26. Rosenfield RL. Clinical review: Adolescent anovulation: maturational mechanisms and implications. The Journal of clinical endocrinology and metabolism. 2013;98(9):3572-83.

27. Hardy TS, Norman RJ. Diagnosis of adolescent polycystic ovary syndrome. Steroids. 2013;78(8):751-4.

28. Rachmiel M, Kives S, Atenafu E, Hamilton J. Primary amenorrhea as a manifestation of polycystic ovarian syndrome in adolescents: a unique subgroup? Arch Pediatr Adolesc Med. 2008;162(6):521-5.

29. Jeffrey Chang R, Coffler MS. Polycystic ovary syndrome: early detection in the adolescent. Clinical obstetrics and gynecology. 2007;50(1):178-87.

30. Olutunmbi Y, Paley K, English JC, 3rd. Adolescent female acne: etiology and management. Journal of pediatric and adolescent gynecology. $2008 ; 21(4): 171-6$.

31. Plouffe L, Jr. Disorders of excessive hair growth in the adolescent. Obstet Gynecol Clin North Am. 2000;27(1):79-99. 
32. Biro FM, Emans SJ. Whither PCOS? The challenges of establishing hyperandrogenism in adolescent girls. The Journal of adolescent health : official publication of the Society for Adolescent Medicine. 2008;43(2):103-5.

33. Diamanti-Kandarakis E. PCOS in adolescents. Best practice \& research Clinical obstetrics \& gynaecology. 2010;24(2):173-83.

34. Venturoli S, Porcu E, Fabbri R, Pluchinotta V, Ruggeri S, Macrelli S, et al. Longitudinal change of sonographic ovarian aspects and endocrine parameters in irregular cycles of adolescence. Pediatric research. 1995;38(6):974-80.

35. Balen AH, Laven JS, Tan SL, Dewailly D. Ultrasound assessment of the polycystic ovary: international consensus definitions. Human reproduction update. $2003 ; 9(6): 505-14$

36. Mortensen M, Rosenfield RL, Littlejohn E. Functional significance of polycystic-size ovaries in healthy adolescents. The Journal of clinical endocrinology and metabolism. 2006;91(10):3786-90.

37. Fruzzetti F, Campagna AM, Perini D, Carmina E. Ovarian volume in normal and hyperandrogenic adolescent women. Fertility and sterility. 2015;104(1):196-9.

38. Herter LD, Magalhaes JA, Spritzer PM. Relevance of the determination of ovarian volume in adolescent girls with menstrual disorders. Journal of clinical ultrasound : JCU. 1996;24(5):243-8.

39. Carmina E, Oberfield SE, Lobo RA. The diagnosis of polycystic ovary syndrome in adolescents. American journal of obstetrics and gynecology. 2010;203(3):201 e1-5. 
40. Senaldi L, Gopi RP, Milla S, Shah B. Is ultrasound useful in the diagnosis of adolescents with polycystic ovary syndrome? Journal of pediatric endocrinology \& metabolism : JPEM. 2015;28(5-6):605-12.

41. Shah B, Parnell L, Milla S, Kessler M, David R. Endometrial thickness, uterine, and ovarian ultrasonographic features in adolescents with polycystic ovarian syndrome. Journal of pediatric and adolescent gynecology. 2010;23(3):146-52.

42. Ibanez L, Potau N, Francois I, de Zegher F. Precocious pubarche, hyperinsulinism, and ovarian hyperandrogenism in girls: relation to reduced fetal growth. The Journal of clinical endocrinology and metabolism. 1998;83(10):3558-62.

43. Ibanez L, Potau N, Virdis R, Zampolli M, Terzi C, Gussinye M, et al. Postpubertal outcome in girls diagnosed of premature pubarche during childhood: increased frequency of functional ovarian hyperandrogenism. The Journal of clinical endocrinology and metabolism. 1993;76(6):1599-603.

44. Damiani D, Kuba VM, Cominato L, Damiani D, Dichtchekenian V, Menezes Filho HC. [Metabolic syndrome in children and adolescents: doubts about terminology but not about cardiometabolic risks]. Arquivos brasileiros de endocrinologia e metabologia. 2011;55(8):576-82.

45. Libman I, Arslanian SA. Type II diabetes mellitus: no longer just adults. Pediatric annals. 1999;28(9):589-93.

46. Type 2 diabetes in children and adolescents. American Diabetes Association. Diabetes care. 2000;23(3):381-9. 
47. Zawadsky J DA. Diagnostic criteria for polycystic ovary syndrome.

Polycystic Ovary Syndrome: Dunaif A, Givens JR, Haseltine FP, Merriam GR, 1992. p. 377-84.

48. Rotterdam EA-SPCWG. Revised 2003 consensus on diagnostic criteria and long-term health risks related to polycystic ovary syndrome. Fertility and sterility. $2004 ; 81(1): 19-25$.

49. Azziz R, Carmina E, Dewailly D, Diamanti-Kandarakis E, EscobarMorreale HF, Futterweit W, et al. The Androgen Excess and PCOS Society criteria for the polycystic ovary syndrome: the complete task force report. Fertility and sterility. 2009;91(2):456-88.

50. Witchel SF, Oberfield S, Rosenfield RL, Codner E, Bonny A, Ibanez L, et al. The Diagnosis of Polycystic Ovary Syndrome during Adolescence. Hormone research in paediatrics. 2015.

51. Sultan C, Paris F. Clinical expression of polycystic ovary syndrome in adolescent girls. Fertility and sterility. 2006;86 Suppl 1:S6.

52. Christensen SB, Black MH, Smith N, Martinez MM, Jacobsen SJ, Porter $\mathrm{AH}$, et al. Prevalence of polycystic ovary syndrome in adolescents. Fertility and sterility. 2013;100(2):470-7.

53. Vural B, Caliskan E, Turkoz E, Kilic T, Demirci A. Evaluation of metabolic syndrome frequency and premature carotid atherosclerosis in young women with polycystic ovary syndrome. Human reproduction. 2005;20(9):2409-13.

54. Tsikouras P, Spyros L, Manav B, Zervoudis S, Poiana C, Nikolaos T, et al. Features of Polycystic Ovary Syndrome in adolescence. J Med Life. $2015 ; 8(3): 291-6$. 
55. Khan U. Polycystic ovary syndrome in adolescents. Journal of pediatric and adolescent gynecology. 2007;20(2):101-4.

56. Azziz R, Hincapie LA, Knochenhauer ES, Dewailly D, Fox L, Boots LR. Screening for 21-hydroxylase-deficient nonclassic adrenal hyperplasia among hyperandrogenic women: a prospective study. Fertility and sterility. 1999;72(5):915-25.

57. Boguszewski MC, Mericq V, Bergada I, Damiani D, Belgorosky A, Gunczler P, et al. Latin American consensus: children born small for gestational age. BMC pediatrics. 2011;11:66.

58. van Hooff MH, van der Meer M, Lambalk CB, Schoemaker J. Variation of luteinizing hormone and androgens in oligomenorrhoea and its implications for the study of polycystic ovary syndrome. Human reproduction. 1999;14(7):16849.

59. Lujan ME, Jarrett BY, Brooks ED, Reines JK, Peppin AK, Muhn N, et al. Updated ultrasound criteria for polycystic ovary syndrome: reliable thresholds for elevated follicle population and ovarian volume. Human reproduction. 2013;28(5):1361-8.

60. Palmert MR, Gordon CM, Kartashov AI, Legro RS, Emans SJ, Dunaif A. Screening for abnormal glucose tolerance in adolescents with polycystic ovary syndrome. The Journal of clinical endocrinology and metabolism. 2002;87(3):1017-23.

61. National High Blood Pressure Education Program Working Group on High Blood Pressure in C, Adolescents. The fourth report on the diagnosis, evaluation, and treatment of high blood pressure in children and adolescents. Pediatrics. 2004;114(2 Suppl 4th Report):555-76. 
62. Fernandez JR, Redden DT, Pietrobelli A, Allison DB. Waist circumference percentiles in nationally representative samples of AfricanAmerican, European-American, and Mexican-American children and adolescents. The Journal of pediatrics. 2004;145(4):439-44.

63. Kuba VM, Leone C, Damiani D. Is waist-to-height ratio a useful indicator of cardio-metabolic risk in 6-10-year-old children? BMC pediatrics. 2013;13:91. 64. World Health Organization (WHO) [Available from: http://www.who.int/growthref/who2007 bmi for age/en/.

65. Bussab WOM, P.A. Estatística Básica. ed. a, editor. São Paulo: Saraiva; 2006.

66. Team RC. A language and environment for statistical computing. Viena, Austria: R Foundation for Statistical Computing; 2015.

67. de Faria FR, Gusmao LS, Faria ER, Goncalves VS, Cecon RS, Franceschini SD, et al. Polycystic ovary syndrome and intervening factors in adolescents from 15 to 18 years old. Rev Assoc Med Bras. 2013;59(4):341-6. 68. Joshi B, Mukherjee S, Patil A, Purandare A, Chauhan S, Vaidya R. A cross-sectional study of polycystic ovarian syndrome among adolescent and young girls in Mumbai, India. Indian journal of endocrinology and metabolism. 2014;18(3):317-24.

69. Motta AB. The role of obesity in the development of polycystic ovary syndrome. Current pharmaceutical design. 2012;18(17):2482-91.

70. Geier LM, Bekx MT, Connor EL. Factors contributing to initial weight loss among adolescents with polycystic ovary syndrome. Journal of pediatric and adolescent gynecology. 2012;25(6):367-70. 
71. Ollila MM, Piltonen T, Puukka K, Ruokonen A, Jarvelin MR, Tapanainen JS, et al. Weight Gain and Dyslipidemia in Early Adulthood Associate With Polycystic Ovary Syndrome: Prospective Cohort Study. The Journal of clinical endocrinology and metabolism. 2016;101(2):739-47.

72. West S, Lashen H, Bloigu A, Franks S, Puukka K, Ruokonen A, et al. Irregular menstruation and hyperandrogenaemia in adolescence are associated with polycystic ovary syndrome and infertility in later life: Northern Finland Birth Cohort 1986 study. Human reproduction. 2014;29(10):2339-51.

73. Glueck CJ, Woo JG, Khoury PR, Morrison JA, Daniels SR, Wang P. Adolescent oligomenorrhea (age 14-19) tracks into the third decade of life (age 20-28) and predicts increased cardiovascular risk factors and metabolic syndrome. Metabolism. 2015;64(4):539-53.

74. Bouzas IC, Cader SA, Leao L, Kuschnir MC, Braga C. Menstrual cycle alterations during adolescence: early expression of metabolic syndrome and polycystic ovary syndrome. Journal of pediatric and adolescent gynecology. $2014 ; 27(6): 335-41$.

75. Yoo RY, Dewan A, Basu R, Newfield R, Gottschalk M, Chang RJ. Increased luteinizing hormone pulse frequency in obese oligomenorrheic girls with no evidence of hyperandrogenism. Fertility and sterility. 2006;85(4):104956.

76. Rosenfield RL, Ehrmann DA, Littlejohn EE. Adolescent polycystic ovary syndrome due to functional ovarian hyperandrogenism persists into adulthood. The Journal of clinical endocrinology and metabolism. 2015;100(4):1537-43. 
77. Youngster M, Ward VL, Blood EA, Barnewolt CE, Emans SJ, Divasta AD. Utility of ultrasound in the diagnosis of polycystic ovary syndrome in adolescents. Fertility and sterility. 2014;102(5):1432-8.

78. Amiel SA, Sherwin RS, Simonson DC, Lauritano AA, Tamborlane WV. Impaired insulin action in puberty. A contributing factor to poor glycemic control in adolescents with diabetes. The New England journal of medicine. 1986;315(4):215-9.

79. Vilmann LS, Thisted E, Baker JL, Holm JC. Development of obesity and polycystic ovary syndrome in adolescents. Hormone research in paediatrics. 2012;78(5-6):269-78.

80. Roe AH, Prochaska E, Smith M, Sammel M, Dokras A. Using the androgen excess-PCOS society criteria to diagnose polycystic ovary syndrome and the risk of metabolic syndrome in adolescents. J Pediatr. 2013;162(5):93741.

81. Franks S. Polycystic ovary syndrome. The New England journal of medicine. 1995;333(13):853-61.

82. Essah PA, Wickham EP, Nestler JE. The metabolic syndrome in polycystic ovary syndrome. Clinical obstetrics and gynecology. 2007;50(1):20525.

83. Amato MC, Guarnotta V, Forti D, Donatelli M, Dolcimascolo S, Giordano C. Metabolically healthy polycystic ovary syndrome (MH-PCOS) and metabolically unhealthy polycystic ovary syndrome (MU-PCOS): a comparative analysis of four simple methods useful for metabolic assessment. Human reproduction. 2013;28(7):1919-28. 
84. Leibel NI, Baumann EE, Kocherginsky M, Rosenfield RL. Relationship of adolescent polycystic ovary syndrome to parental metabolic syndrome. The Journal of clinical endocrinology and metabolism. 2006;91(4):1275-83.

85. Rahmanpour H, Jamal L, Mousavinasab SN, Esmailzadeh A, Azarkhish K. Association between polycystic ovarian syndrome, overweight, and metabolic syndrome in adolescents. Journal of pediatric and adolescent gynecology. 2012;25(3):208-12.

86. Coviello AD, Legro RS, Dunaif A. Adolescent girls with polycystic ovary syndrome have an increased risk of the metabolic syndrome associated with increasing androgen levels independent of obesity and insulin resistance. The Journal of clinical endocrinology and metabolism. 2006;91(2):492-7.

87. Knowler WC, Barrett-Connor E, Fowler SE, Hamman RF, Lachin JM, Walker EA, et al. Reduction in the incidence of type 2 diabetes with lifestyle intervention or metformin. The New England journal of medicine. 2002;346(6):393-403.

88. Ibanez L, Valls C, Ferrer A, Marcos MV, Rodriguez-Hierro F, de Zegher F. Sensitization to insulin induces ovulation in nonobese adolescents with anovulatory hyperandrogenism. The Journal of clinical endocrinology and metabolism. 2001;86(8):3595-8.

89. Spritzer PM, Motta AB. Adolescence and polycystic ovary syndrome: current concepts on diagnosis and treatment. Int J Clin Pract. $2015 ; 69(11): 1236-46$.

90. Morris S, Grover S, Sabin MA. What does a diagnostic label of 'polycystic ovary syndrome' really mean in adolescence? a review of current practice recommendations. Clin Obes. 2016;6(1):1-18. 
